\title{
Abl2:Cortactin Interactions Regulate Dendritic Spine Stability via Control of a Stable Filamentous Actin Pool
}

\author{
${ }^{\circ}$ Juliana E. Shaw, ${ }^{1}$ Michaela B.C. Kilander, ${ }^{4}{ }^{\circ}$ Yu-Chih Lin, ${ }^{1,4}$ and ${ }^{\circledR}$ Anthony J. Koleske ${ }^{1,2,3}$ \\ ${ }^{1}$ Departments of Molecular Biophysics and Biochemistry, ${ }^{2}$ Neuroscience, ${ }^{3}$ Interdepartmental Neuroscience Program, Yale University, New Haven, \\ Connecticut 06520, and ${ }^{4}$ Program in Neuroscience, Hussman Institute for Autism, Baltimore, Maryland 21201
}

Dendritic spines act as the receptive contacts at most excitatory synapses. Spines are enriched in a network of actin filaments comprised of two kinetically distinct pools. The majority of spine actin is highly dynamic and regulates spine size, structural plasticity, and postsynaptic density organization. The remainder of the spine actin network is more stable, but the function of this minor actin population is not well understood, as tools to study it have not been available. Previous work has shown that disruption of the Abl2/Arg nonreceptor tyrosine kinase in mice compromises spine stability and size. Here, using cultured hippocampal neurons pooled from both sexes of mice, we provide evidence that binding to cortactin tethers Abl2 in spines, where Abl2 and cortactin maintain the small pool of stable actin required for dendritic spine stability. Using fluorescence recovery after photobleaching of GFP-actin, we find that disruption of Abl2:cortactin interactions eliminates stable actin filaments in dendritic spines, significantly reducing spine density. A subset of spines remaining after Abl2 depletion retain their stable actin pool and undergo activity-dependent spine enlargement, associated with increased cortactin and GluN2B levels. Finally, tonic increases in synaptic activity rescue spine loss following Abl2 depletion by promoting cortactin enrichment in vulnerable spines. Together, our findings strongly suggest that Abl2:cortactin interactions promote spine stability by maintaining pools of stable actin filaments in spines.

Key words: Abl2; actin; cortactin; dendritic spine stability; FRAP; stable actin

Significance Statement

Dendritic spines contain two kinetically distinct pools of actin. The more abundant, highly dynamic pool regulates spine shape, size, and plasticity. The function of the smaller, stable actin network is not well understood, as tools to study it have not been available. We demonstrate here that $\mathrm{Abl} 2$ and its substrate and interaction partner, cortactin, are essential to maintain the stable pool in spines. Depletion of the stable actin pool via disruption of Abl2 or cortactin, or interactions between the proteins, significantly reduces spine stability. We also provide evidence that tonic increases in synaptic activity promote spine stability via enrichment of cortactin in spines, suggesting that synaptic activity acts on the stable actin pool to stabilize dendritic spines.

\section{Introduction}

Dendritic spines serve as receptive postsynaptic compartments on excitatory neurons and defects in their formation, density,

\footnotetext{
Received Sep. 21, 2020; revised Jan. 15, 2021; accepted Feb. 16, 2021.

Author contributions: J.E.S., Y.-C.L., and A.J.K. designed research; J.E.S., M.B.C.K., and Y.-C.L. performed research; J.E.S., M.B.C.K., and Y.-C.L. analyzed data; J.E.S. wrote the first draft of the paper; J.E.S., Y.-C.L., and A.J.K. edited the paper; J.E.S. and A.J.K. wrote the paper.

This work was supported by National Institutes of Health Grants NS089662 to A.J.K., NS105640 to A.J.K. and Michael J. Higley, MH115939 to A.J.K., and F31 MH116571 to J.E.S. J.E.S. was supported by National Institutes of Health Training Grant T32GM007223 to Susan J. Baserga. We thank Aaron Levy, Josie Bircher, Melissa Carrizales, and Amanda Jeng for helpful comments on this manuscript; and other members of the A.J.K. laboratory for critical discussion and suggestions.

The authors declare no competing financial interests.

Correspondence should be addressed to Anthony J. Koleske at anthony.koleske@yale.edu or Yu-Chih Lin at yclin@hussmanautism.org.

https://doi.org/10.1523/JNEUROSCI.2472-20.2021

Copyright $\odot 2021$ the authors
}

and shape are hallmarks of many brain disorders (Fiala et al., 2002; Penzes et al., 2011). Spine shape and stability are principally supported by a filamentous actin network, which also organizes scaffolding proteins and neurotransmitter receptors at the postsynaptic density (Fischer et al., 1998; Alvarez and Sabatini, 2007; Schubert and Dotti, 2007; W. H. Lin and Webb, 2009; Hotulainen and Hoogenraad, 2010). Spine actin filaments assemble into higher-order structures that undergo dynamic rearrangements and provide the forces to drive changes in spine shape and size (Matus, 2000; Luo, 2002; Sekino et al., 2007; Chazeau and Giannone, 2016). Indeed, molecules that regulate spine plasticity and stability, including cell surface receptors and kinases, often converge on signaling pathways that control polymerization, stability, and contractility of actin networks. Therefore, the cytoskeletal machinery is a key module to regulate spine plasticity and stability. 
Fluorescence recovery after photobleaching (FRAP) experiments of GFP-actin have determined that most (80\%-85\%) spine actin filaments are dynamic, undergoing ongoing polymerization and turnover within 10s of seconds, while the remainder of actin is more stable. These distinct kinetic pools of actin likely play unique roles in the spine. Dynamic filaments mediate a net retrograde flow of actin from the spine periphery toward the spine core and are perfectly positioned to promote changes in spine size and shape (Honkura et al., 2008; Frost et al., 2010) and also regulate neurotransmitter receptor localization or gating properties (Rosenmund and Westbrook, 1993; Borgdorff and Choquet, 2002; Kerr and Blanpied, 2012). Photoactivation of mEOS3.2actin reveals that the small, stable pool of actin is enriched at the spine base and turns over much more slowly ( $\mathrm{t} \sim 17 \mathrm{~min}$ ) (Honkura et al., 2008). The relative proportion of stable actin increases during synapse maturation (Koskinen et al., 2014) and may serve as a base for polymerization of new actin during spine enlargement (Mikhaylova et al., 2018). The functions of the stable actin pool have been largely understudied, primarily because we lack an in-depth understanding of the molecules and mechanisms that regulate it.

The Abl2/Arg nonreceptor tyrosine kinase interacts with its substrate and binding partner cortactin to regulate actin-based structures in many cellular contexts (MacGrath and Koleske, 2012a; Schnoor et al., 2018). Both proteins localize to dendritic spines where they promote spine stability (Hering and Sheng, 2003; Iki et al., 2005; Sfakianos et al., 2007; Y. C. Lin et al., 2013; MacGillavry et al., 2016; Mikhaylova et al., 2018). Abl2 binds actin filaments cooperatively (Wang et al., 2001) and increases the binding stoichiometry of cortactin for actin filaments (MacGrath and Koleske, 2012b). These interactions significantly impact actin filament stability: at saturated binding, Abl2 and cortactin each stabilize actin filaments (Courtemanche et al., 2015; Scherer et al., 2018), but mixtures of Abl2 and cortactin at concentrations far below saturated binding also stabilize actin filaments (MacGrath and Koleske, 2012b; Courtemanche et al., 2015). While Abl2 and cortactin synergize to stabilize actin filaments in vitro, it is unclear whether or how they contribute to actin stability in neurons or how this mechanism might impact dendritic spine shape or stability.

Here, we show that cortactin anchors Abl2 in dendritic spines via an SH2:phosphotyrosine interface, and mutational disruption of this interface dislodges Abl2 from spines. Depletion of either protein eliminates the stable actin pool in spines, as measured by FRAP of GFP-actin. Disruption of this stable pool correlates with a significant loss of dendritic spines. We find that a subset of spines remaining after Abl2 depletion exhibit increased spine head widths, which is tightly associated with increased cortactin and GluN2B levels, and retention of the stable actin pool. Finally, tonic increases in activity can rescue spine loss following Abl2 depletion by promoting cortactin enrichment in vulnerable spines that are otherwise lost in basal conditions. Together, our data indicate that Abl2 and cortactin synergize to maintain dendritic spine stable actin, which is critical for spine stability.

\section{Materials and Methods}

Animals. All animal procedures were compliant with federal regulations and approved by the Animal Care and Use Committees at Yale University, University of Maryland at Baltimore, and Hussman Institute for Autism. BALB/c mice were purchased from Charles River Laboratories. Animals were housed and cared for in a Yale-sponsored Office for Protection from Research Risks-approved animal facility. Neonatal mice of both sexes were used for neuronal culture preparation.
Cell culture and transfections. HEK293 cells were plated in 6-well plates and maintained in high glucose DMEM (Invitrogen) growth media supplemented with $1 \%$ penicillin/streptomycin (Invitrogen), 2 mM L-glutamine (Invitrogen), and 10\% FBS (Sigma Millipore). Primary neuronal cultures were prepared from postnatal day 1 mouse hippocampus as previously described (Y. C. Lin et al., 2013). Neurons were maintained in serum-free media containing $1 \%$ penicillin/streptomycin, $2 \mathrm{~mm}$ L-glutamine, and 2\% B27-supplement (Invitrogen) in Neurobasal media (NB-SFM) (Invitrogen). Glass coverslips in 24-well plates were coated with $20 \mu \mathrm{g} / \mathrm{ml}$ of poly-(D)-lysine (Corning) overnight at $37^{\circ} \mathrm{C}$ and $1 \mu \mathrm{g} /$ $\mathrm{ml}$ laminin 111 (Corning) for $2 \mathrm{~h}$ at $37^{\circ} \mathrm{C}$. Neurons were plated at a cell density of $0.3 \times 10^{6} \mathrm{cell} /$ well. Transfection was performed using a calcium-phosphate precipitation method in both HEK293 cells and primary neurons. TTX citrate ( $1 \mu \mathrm{M}$; Tocris Bioscience), $20 \mu \mathrm{m}$ bicuculine methiodide (Sigma Millipore), and $20 \mu \mathrm{M}$ CNQX disodium salt (Millipore Sigma) were added directly to the culture media immediately following transfection and incubated with neurons $72 \mathrm{~h}$ before fixation.

Plasmids. WT and Abl2 mutants were constructed in N1-mRFP plasmid as previously described (A. L. Miller et al., 2004; Lapetina et al., 2009; Peacock et al., 2010). The shRNA-resistant mutations (Y. C. Lin et al., 2013) were introduced in WT-Abl2, Abl2-KI, Abl2 N-term, and Abl2-R198K mutants and subcloned into shAbl2 plasmid for the experiment shown in Figure 1. The shRNA targeted to mouse cortactin was generated by synthesizing two complementary oligonucleotides (shcort\#1: $\quad 5^{\prime}$-tgcactgctcacaagtggacttcaagagagtccacttgtgagcagtgctttttc$3^{\prime}$ and shcort\#2: $5^{\prime}$-tcgagaaaaaagcactgctcacaagtggactctcttgaagtccacttgtgagcagtgca-3') and cloned into the pLL3.7 vector (Rubinson et al., 2003) between HpaI and XhoI sites. Three tyrosine sites at 421, 466, and 482 were mutated to phenylalanine to make 3YFcortactin mutant as described previously (Lapetina et al., 2009). The shRNA-resistant cortactin cort ${ }^{\mathrm{r}}$-RFP, 3YFcort ${ }^{\mathrm{r}}$-RFP, and W525Acort ${ }^{\mathrm{r}}$-RFP constructs were made by mutating three nucleotides in the shcort recognition sequences with two PCR primers: $5^{\prime}$-cgaagctttccaagcactgctctcaagtcgactccgtccggg- $3^{\prime}$ and $5^{\prime}$-ccgaagcccogacggagtcgacttgagagcagtgcttgg- $3^{\prime}$.

Western blot analysis. Cells were harvested then lysed in buffer containing $20 \mathrm{~mm}$ Tris, $\mathrm{pH}$ 7.5, $150 \mathrm{~mm} \mathrm{NaCl}, 2$ mм EDTA, 1\% Triton X100 , and phosphatase and protease inhibitor cocktails. Protein concentration was determined using a Pierce BCA Protein assay Kit (Thermo Scientific); $75 \mu \mathrm{g}$ of total protein from HEK293 cell lysates was used in immunoblots to determine the knockdown efficiency. Protein samples were run on a $10 \%$ SDS-PAGE and transferred to nitrocellulose membranes for immunoblotting with antibodies against RFP (Invitrogen) and $\beta$-actin (Abcam) in TBST containing 3\% milk. Densitometry of blotted protein was performed on scanned films using ImageJ (National Institutes of Health).

Immunocytochemistry and morphometric analysis. Neurons were fixed with $4 \%$ PFA in PBS and permeabilized with $0.1 \%$ Triton X-100 and 3\% BSA in PBS. Cells were immunostained with primary antibodies against GFP (Rockland) or RFP (Chemicon) followed by Alexa- 488 and -594-conjugated secondary antibodies (Invitrogen). For total filamentous actin staining with fluorescent-phalloidin, neurons were fixed with 2\% PFA in cytoskeleton buffer (10 mм MES, pH 6.8, $138 \mathrm{~mm} \mathrm{KCl,} 3 \mathrm{~mm}$ $\mathrm{MgCl}_{2}, 2 \mathrm{~mm}$ EGTA, and $0.32 \mathrm{~mm}$ sucrose). Fixed cells were permeabilized with $0.3 \%$ Triton X-100/TBS ( $150 \mathrm{~mm} \mathrm{NaCl}, 20 \mathrm{~mm}$ Tris, pH 7.4) and blocked with $0.1 \%$ Triton X-100/2\% BSA/10\% Normal Donkey Serum/TBS. Cells were incubated at $4^{\circ} \mathrm{C}$ overnight with anti-RFP (Rockland). The cells were then sequentially incubated with Alexa-594conjugated secondary antibodies (Invitrogen) and Atto647N-phalloidin (Sigma Millipore). For GluN2B staining, neurons were fixed with $2 \%$ PFA in cytoskeleton buffer and then incubated with the GluN2B antibody (Alomone Labs) for $2 \mathrm{~h}$ at room temperature. Cells were then permeabilized, blocked, and incubated with an anti-EGFP antibody (Rockland), as described above. For dendritic spine analysis, 2-3 dendrite segments per neuron were selected randomly from secondary or tertiary branches on the apical dendrite for imaging. Images were taken using a LSM710 confocal microscope with $63 \times$ NA 1.4 objective and $4 \times$ zoom. Dendritic spine density was quantified using ImageJ (National Institutes of Health). Quantified dendrite segments were averaged for each neuron analyzed, and each averaged dendritic spine density for a cell is 
A
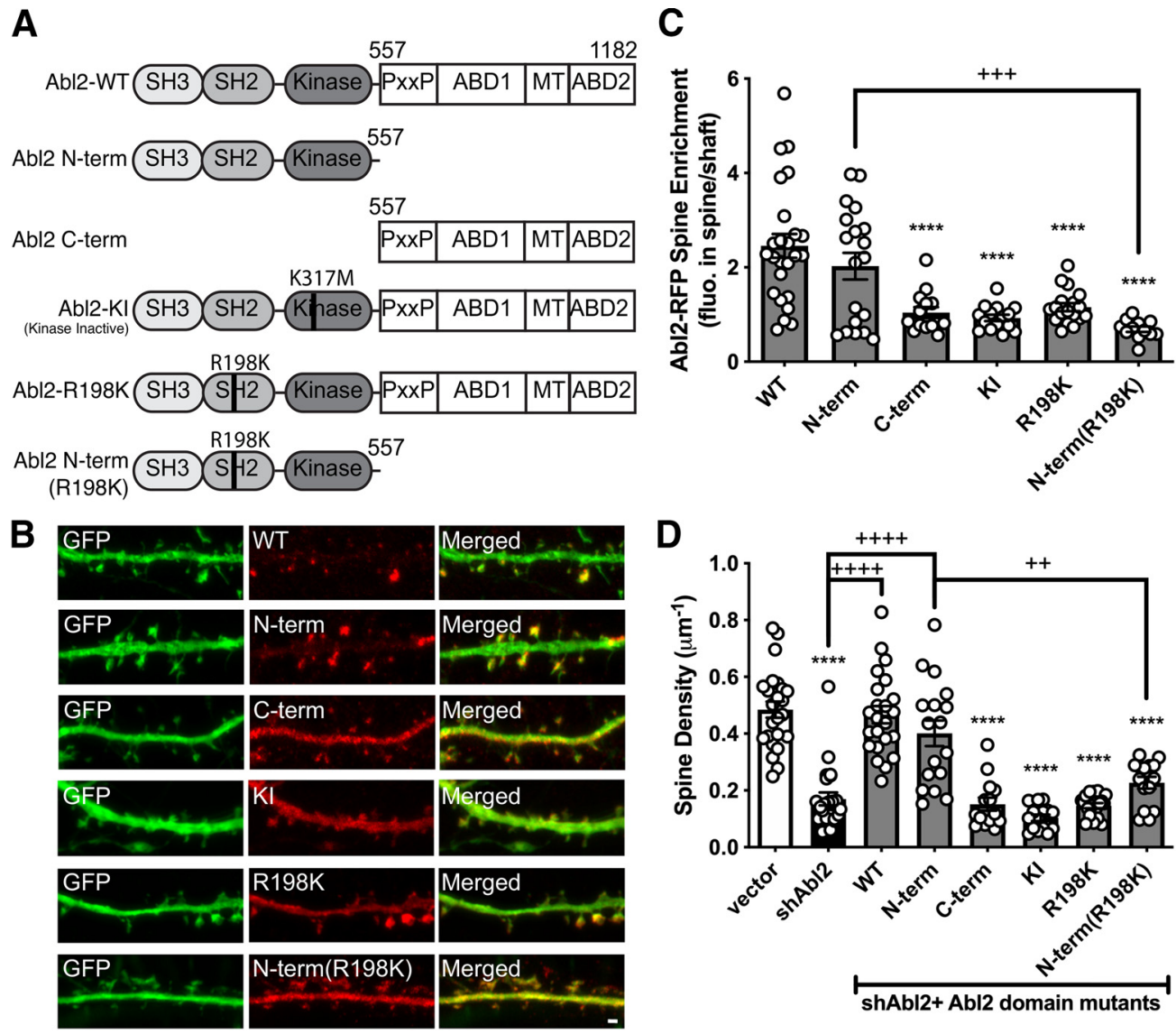

Figure 1. Abl2 kinase and SH2 domains are required for spine localization and spine stability. $\boldsymbol{A}$, Illustration of Abl2 domain mutants used in the experiment. $\boldsymbol{B}$, Representative confocal images of neurons cotransfected with GFP and RFP-tagged Abl2 or Abl2 domain. Scale bar, $1 \mu \mathrm{m}$. C, The relative fluorescence intensity (spine enrichment) of Abl2-RFP or Abl2 domain mutants in the dendritic spine versus dendrite shaft. Spine enrichment $>1$ indicates a preferential localization to dendritic spines. Data are mean \pm SEM $\left(n=12-25\right.$ neurons/group). ${ }^{+++} p<0.001$; $* * * * p<0.0001$; ordinary one-way ANOVA with Tukey multiple comparisons test. $\boldsymbol{D}$, Dendritic spine density of neurons transfected with empty vector control, shAbl2, or shAbl2 with Abl2/ Abl2 domain mutants. Only neurons transfected with Abl2 domain mutants that contain a functional kinase domain and an SH2 domain (e.g., Abl2-WT and Abl2 N-term) show spine density comparable with control neurons. Data are mean \pm SEM ( $n=14-24$ neurons/group). ${ }^{++} p<0.01 ; * * * *{ }_{1}^{++++} p<0.0001$; ordinary one-way ANOVA with Tukey multiple comparisons test.

represented as a sample $(n)$ in the graphs. To measure the spine enrichment of cortactin and Abl2 content, a maximum projection was obtained for the $z$ stack of the dendrite segment. An ROI was selected in the dendritic spine, adjacent dendrite shaft, and over a region in the background. Spine enrichment was quantified using the following equation:

$$
\text { Spine enrichment }=\frac{\left(R_{\text {spine }}-R_{\text {sol }} \text { background }\right)}{\left(R_{\text {shaft }}-R_{\text {background }}\right)}
$$

Spine enrichment was quantified for 4-6 spines per neuron; then an average was taken for the cell. The average spine enrichment for a neuron is represented as a sample $(n)$ in the graphs.

FRAP. Dissociated P0-1 mouse hippocampal neurons were plated onto $35 \mathrm{~mm}$ glass-bottom dishes (MatTek). Cells were transfected using a calcium-phosphate precipitation method at 12 DIV with a 1:1 ratio of pN1-EGFP-human $\beta$-actin to pLL3.7-shRNA-mRFP plasmids targeting either Abl2 or cortactin. Live-cell FRAP imaging of pyramidal neurons in NB-SFM without phenol red culture medium was performed at 15 DIV (72 h after transfection, when we see net spine destabilization in Abl2 KD neurons (Y. C. Lin et al., 2013)) at $37^{\circ} \mathrm{C}$ using a $60 \times$ oil immersion objective on an UltraVIEW VoX (PerkinElmer) spinning disk confocal microscope equipped with the PhotoKinesis (PK) Device. Volocity software (Improvision) was used to select the bleach ROI over the head of a spine on the secondary or tertiary branch of the apical dendrite. Prebleach fluorescence intensities were averaged and normalized to 1 , and the curves were fit with two-phase exponential equations, as described with some modifications detailed below (Star et al., 2002; Koskinen et al., 2014). Before bleaching, 6-10 frames were captured at 3 frames per second (fps) then bleaching was performed using the $488 \mathrm{~nm}$ laser at $20 \%$ power with the following Ultraview PK settings: $100 \mathrm{PK}$ cycles, $200 \mathrm{~ms}$ spot period, and $1 \mathrm{PK}$ spot cycle. During the recovery phase images were acquired at $3 \mathrm{fps}$ for $10 \mathrm{~s}$ then for the duration of the experiment at $1 \mathrm{fps}$ to increase sample protection and reduce phototoxicity on the sample. Three to 5 spines/cell were bleached. Background fluorescence was determined in an area outside the cell using a ROI size equal to the bleach ROI. Potential FRAP-unrelated bleaching effects were corrected using a ROI with the same dimensions as the bleach ROI placed in an unbleached area with similar initial fluorescence as the bleach ROI, termed reference spine. Therefore, any photobleaching, unrelated to FRAP, is corrected for using a linear model before fitting the fluorescence recovery curve. Fluorescence recovery (mobile fraction) over time was calculated by the following two-phase exponential equation:

$$
y=b * c *\left(1-e^{\left(-k_{1}\left(x-x_{\text {lag }}\right)\right)}\right)+b *(1-c) *\left(1-e^{\left(-k_{2}\left(x-x_{\text {lag }}\right)\right)}\right)
$$

where $b$ is the plateau, $c$ is the fraction of dynamic actin, $k_{1}$ and $k_{2}$ are rate constants with the units inverse seconds, and $x_{\text {lag }}$ is the time immediately following bleaching. The stable actin fraction is then defined as $1-b$ and $\tau$, the time constant for the dynamic actin turnover, is defined as $1 / k_{1}$ and has units of seconds. Abl2 FRAP was fit to a one-phase exponential equation that was modified from the above equation by excluding the second term. 
Spine head width measurement. Spine head width was measured in ImageJ using methods previously published with some modifications (Nagerl et al., 2008; Adrian et al., 2017). In short, spine head width was measured by drawing a line on the short axis of the spine. The fluorescence intensity profile was then plotted and fit to the following Gaussian curve:

$$
y=a+(b-a) e^{-\frac{(x-c)^{2}}{2 \sigma^{2}}}
$$

where $a$ is the baseline intensity, $b$ is the curve's peak intensity, $c$ is the position of the center of the peak, and $\sigma$ is the SD. FWHM was then defined as $2.35 * \sigma$. For spine head width measurements from live samples, the FWHM was averaged from all prebleached frames to account for local drifts of the spine in the focal plane.

Experimental design and statistical analysis. All data were from at least three independent neuronal cultures per condition. Imaging and analyses were performed while blinded to experimental conditions. Statistical analyses were performed in GraphPad Prism 8 or R software. Specific details on $n$ and statistical tests are included in the figure legends. "Control" refers to neurons transfected with a scrambled shRNA expressing soluble fluorescent protein and parts 1 and 2 of a given graph represents the same control datasets, shown in each case as for comparison. Significance was defined by a $p$ value $<0.05$ and a "*" symbol is always used for comparisons to the control group of the dataset. All pooled data are represented as mean \pm SEM. Normality of data were checked with a D'Agostino and Pearson normality test where appropriate. In cases with simple genetic manipulations, an unpaired $t$ test was used. To analyze the effects of knockdown and rescue experiments where many manipulations were performed, we used one-way ANOVA and corrected for multiple comparisons to test for differences between groups. We performed a Pearson correlation test to test for a relationship between cortactin or GluN2B spine enrichment and spine head width. Specific statistical details for each experiment are reported in the figure legends.

\section{Results}

\section{Abl2 kinase activity and $\mathrm{SH} 2$ domains are critical for $\mathrm{Abl} 2$} enrichment in dendritic spines

Abl2 is a large multidomain protein that contains N-terminal Src homology (SH) 3, SH2, and kinase domains, followed by a C-terminal extension that contains a proline-rich PXXP segment, and two F-actin binding domains flanking a microtubule-binding domain (Fig. 1A). When expressed in WT neurons, Abl2-RFP preferentially localizes to spines. While Abl2 $\mathrm{KD}$ decreases spine density, overexpression does not alter spine density (Y. C. Lin et al., 2013). To study the relative contributions of different Abl2 protein domains to its spine enrichment, we expressed different RFP-tagged Abl2 point and truncation mutants (Fig. 1A) along with GFP as a cell fill (Fig. $1 B, C$ ). Consistent with previous studies (Y. C. Lin et al., 2013), we find that Abl2-RFP is enriched approximately twofold in spines relative to dendritic shafts in control neurons (Fig. $1 B, C$ ). The Abl2 N-terminal half (Abl2 Nterm-RFP), which contains the $\mathrm{SH} 3, \mathrm{SH} 2$, and kinase domains, was enriched in spines to a similar extent as full-length Abl2, whereas the Abl2 C-terminal half (Abl2 C-term-RFP) was not (Fig. 1B,C). A kinase-inactive point mutant of Abl2 (Abl2-KIRFP) exhibited significantly less enrichment (Fig. 1C), supporting a key role for kinase activity in proper localization of Abl2 to dendritic spines. We also mutated the Abl2 $\mathrm{SH} 2$ domain (R198K) to abrogate binding to phospho-tyrosine-containing binding partners (Lapetina et al., 2009; Simpson et al., 2015), both in full-length $\mathrm{Abl} 2$ and in Abl2 N-term (Fig. 1A). While both mutants were present in spines, neither was enriched relative to the dendrite shaft (Fig. $1 B, C$ ). Together, these data
Table 1. Summary of Abl2 and cortactin spine enrichment and spine maintenance

\begin{tabular}{llll}
\hline & $\begin{array}{l}\text { Spine } \\
\text { enrichment }\end{array}$ & $\begin{array}{l}\text { Normal spine } \\
\text { density? }\end{array}$ & Cortactin spine enrichment \\
\hline Abl2 & + & Y & \\
Abl2 KD & - & $\mathrm{N}$ & Reduced \\
Abl2 N-term & + & Y & Normal \\
Abl2 C-term & - & $\mathrm{N}$ & \\
Abl2-Kl & - & $\mathrm{N}$ & \\
Abl2-R198K & - & $\mathrm{N}$ & \\
Abl2 N-term (R198K) & - & $\mathrm{N}$ & \\
\hline & & & Abl2 spine enrichment \\
\hline Cortactin & + & Y & \\
Cort KD & - & $\mathrm{N}$ & Reduced \\
3YFcort & + & $\mathrm{N}$ & Reduced and shows increased \\
& & & turnover \\
W525Acort & - & $\mathrm{N}$ &
\end{tabular}

+ , Nearly WT spine enrichment of the mutant; - , no preferential enrichment in the spine compared with the dendrite shaft.

indicate that $\mathrm{Abl} 2$ requires both its kinase activity and $\mathrm{SH} 2$ domains to support its preferential localization to dendritic spines.

\section{Abl2 kinase activity and $\mathrm{SH} 2$ domains are required for dendritic spine maintenance}

We previously showed that knockdown of Abl2 in hippocampal neurons caused progressive reduction of dendritic spines over 72 $\mathrm{h}$ and enlargement of a subset of remaining spines (Y. C. Lin et al., 2013). These defects were rescued by reexpression of the shRNA-resistant Abl2. We used this complementation assay to determine which domains of $\mathrm{Abl} 2$ are important to maintain normal dendritic spine density (Fig. $1 A$ ). Similar to our previous work (Y. C. Lin et al., 2013), we found that Abl2 KD yielded a $65 \pm 8 \%$ decrease in spine density after $72 \mathrm{~h}$ and the full-length shRNA-resistant Abl2-YFP restored spine density to levels observed in the vector alone control. Reexpression of the kinase-inactive (Abl2-KI-YFP) and SH2 domain mutant (Abl2R198K-YFP) failed to rescue spine density (Fig. 1D), which paralleled their lack of enrichment in dendritic spines (Fig. 1C). Surprisingly, the Abl2 N-term-YFP, which completely lacks the C-terminal actin binding domains, was sufficient to support normal dendritic spine density, whereas the Abl2 C-terminal half (Abl2-C-term-YFP) did not (Fig. 1D). Abl2 constructs that are enriched in dendritic spines (Fig. 1C) support spine densities (Fig. 1D) similar to WT Abl2, whereas mutants that disrupt spine enrichment do not support normal spine density (summarized in Table 1). Hence, Abl2 localization to dendritic spines is critical for spine stability.

\section{Cortactin tethers Abl2 in dendritic spines to support normal dendritic spine density}

Abl2 kinase activity and phosphotyrosine binding by its $\mathrm{SH} 2$ domain are necessary for Abl2 to both localize to dendritic spines and support normal dendritic spine density, but the critical partners for $\mathrm{Abl} 2$ in these processes are unknown. Abl2 and cortactin colocalize to actin-rich protrusions in a variety of cellular contexts and reciprocal interactions between the two proteins have been well characterized (Liu et al., 2012; Y. C. Lin et al., 2013; Gifford et al., 2014; Courtemanche et al., 2015). The cortactin SH3 domain binds to a specific Pro-X-X-Pro-X-XPro motif unique to Abl2 (Lapetina et al., 2009; Liu et al., 2012). Subsequent Abl2-mediated phosphorylation of cortactin on 
A

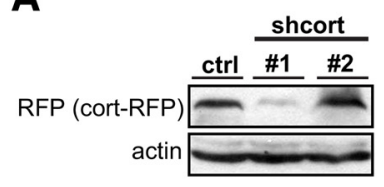

D
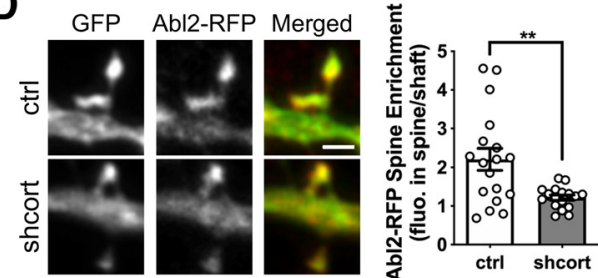

$\mathbf{E}$

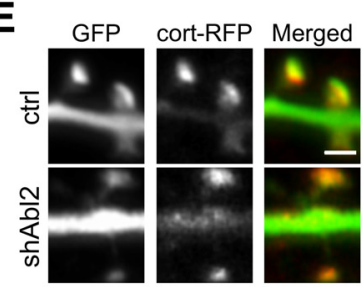

B
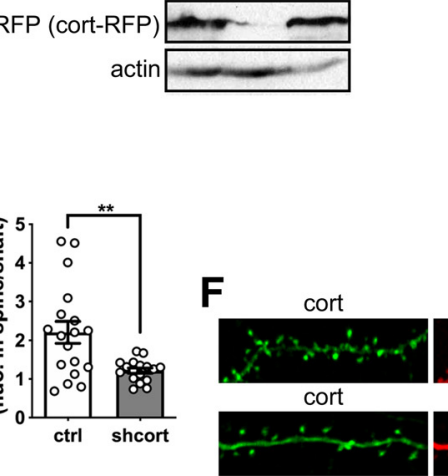

$\mathbf{G}_{\text {t. }}$

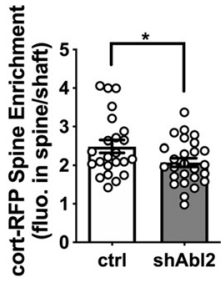

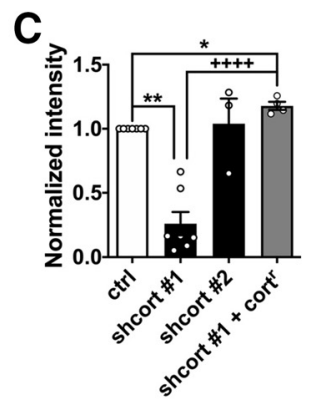
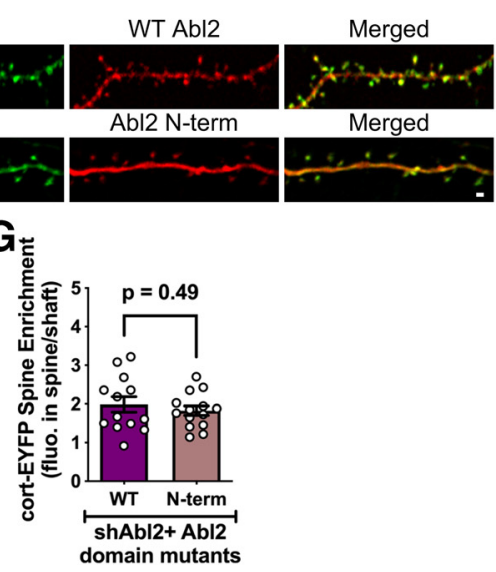

Figure 2. Abl2 enrichment in dendritic spines is strongly dependent on cortactin levels. A, Representative immunoblots of cortactin KD in HEK-293 cells. Two shRNA constructs (shcort \#1 and shcort \#2) were cotransfected with cortactin-RFP in HEK293 cells for $72 \mathrm{~h}$. The expression of cortactin-RFP was determined using RFP antibodies on Western blot analysis. shcort \#1 (later as shcort) has higher KD efficiency than shcort \#2. B, HEK-293 cells were cotransfected with control vector or shcort together with cortactin-RFP or cort'-RFP, an shRNA-resistant form of cortactin, for 72 h. shcort efficiently knocks down cortactin-RFP, but not cort'-RFP. C, Quantification of cortactin-RFP levels indicating shRNA KD of cortactin and resistance of cort'-RFP toward the shRNA. Data are mean \pm SEM ( $n=3-7$ cultures/condition) normalized to the actin signal. $* p<0.05, * * p<0.01,^{++++} p<0.0001$ (one-sample $t$ test). $\boldsymbol{D}$, Representative confocal images and relative enrichment of Abl2-RFP in cortactin KD neurons. Scale bar, $1 \mu \mathrm{m}$. When cortactin is knocked down, Abl2 shows less spine enrichment. Data are mean \pm SEM $(n=17$ or 18 neurons/group). $* * p<0.01$ (unpaired $t$ test). $\boldsymbol{E}$, Representative confocal images and relative enrichment of cort-RFP in Abl2 KD neurons. Scale bar, $1 \mu \mathrm{m}$. Data are mean \pm SEM ( $n=23-$ 26 neurons/group). $* p<0.05$ (unpaired $t$ test). $\boldsymbol{F}$, Representative confocal images of neurons cotransfected with cortactin-EYFP and RFP-tagged Abl2 or Abl2 N-term in Abl2 KD neurons. Scale bar, $1 \mu \mathrm{m}$. G. The relative enrichment of cort-EYFP in dendritic spines of neurons expressing Abl2 or Abl2 N-term. Cortactin enrichment in spines is comparable in each condition. Data are mean $\pm \operatorname{SEM}$ ( $n=13$ or 14 neurons/group). $p=0.49$ (unpaired $t$ test).

tyrosine (Y)421 and Y466 creates binding sites for the Abl2 SH2 domain (Gifford et al., 2014).

We asked whether Abl2 enrichment in spines depends on cortactin, and if so, whether interactions between the proteins are critical for their enrichment. We designed two shRNAs predicted to target cortactin: shcort \#1 and shcort \#2. Transfection of shcort \#1 in HEK-293 cells resulted in a $74 \pm 9 \%$ (difference of means \pm SEM) reduction of a mouse cortactin-RFP fusion protein. shcort \#2 did not impact cortactin-RFP expression (Fig. $2 A, C$ ); therefore, we used shcort \#1 (shcort) to knock down cortactin in subsequent experiments. Levels of an shRNA-resistant cortactin ( $\left.\operatorname{cort}^{\mathrm{r}}-\mathrm{RFP}\right)$, bearing silent mutations that disrupted interaction with shcort \#1, were unchanged up to $72 \mathrm{~h}$ after transfection with shcort \#1 (Fig. 2B,C).

We knocked down Abl2 or cortactin in cultured hippocampal neurons to examine how these manipulations impacted the subcellular localization of its reciprocal binding partner. Consistent with Figure $1 C$ and previous studies, we find that Abl2-RFP and cort-RFP are enriched approximately twofold in dendritic spines relative to shafts in control neurons (Fig. $2 D, E$ ). The enrichment of Abl2-RFP in dendritic spines relative to the dendritic shaft was decreased by $45 \pm 14 \%$ (Fig. $2 D$ ) in cortactin KD neurons, demonstrating that spine enrichment of Abl2 is strongly dependent on the presence of cortactin. These data indicate that cortactin is an important tether for $\mathrm{Abl} 2$ in dendritic spines. In contrast, the relative enrichment of cortactin-RFP in dendritic spines was reduced by $17 \pm 8 \%$ in $\mathrm{Abl} 2 \mathrm{KD}$ neurons (Fig. $2 E$ ) (Y.
C. Lin et al., 2013). Abl2 KD neurons reexpressing full-length Abl2 or Abl 2 N-term also exhibited a twofold enrichment of cort-EYFP in spines (Fig. $2 F, G$ ), an enrichment similar to that observed in control neurons (Fig. $2 E$ ), indicating that cortactin enrichment in spines only partially depends on Abl2.

We next examined the critical determinants in cortactin required for its enrichment in spines and its ability to support Abl2 enrichment in spines. We first measured the enrichment of shRNA-resistant cortactin-RFP and mutant constructs in neurons following treatment with shcort (Fig. $3 A-C$ ). Cortactin bearing nonphosphorylatable tyrosine-to-phenylalanine substitutions at Y421, Y466, and Y482 (3YFcort ${ }^{\mathrm{r}}$-RFP; Fig. 3A) was enriched in spines more than WT cort $^{\mathrm{r}}$-RFP (Fig. 3B,C). Given that cortactin uses its $\mathrm{SH} 3$ domain to engage Abl2 (Lapetina et al., 2009), as well as other synaptic proteins (Chen and Hsueh, 2012; MacGillavry et al., 2016; Mikhaylova et al., 2018), we mutated the conserved tryptophan residue in the cortactin $\mathrm{SH} 3$ domain to alanine (W525A), which disrupts its binding to the Abl2 Pro-X-X-Pro-X-X-Pro motif (Fig. $3 A$ ). When introduced into cortactin KD neurons, the W525Acort ${ }^{\mathrm{r}}$-RFP was depleted from spines compared to WT cort ${ }^{\mathrm{r}}$-RFP (Fig. $3 B, C$ ). Thus, the cortactin $\mathrm{SH} 3$ domain is a major tether for its spine enrichment, whereas mutation of the three tyrosine residues does not result in a loss of cortactin enrichment in spines.

Cortactin KD neurons exhibited a $31 \pm 8 \%$ reduction in spine density (Fig. 3D), a slightly larger reduction to that previously reported (Hering and Sheng, 2003). Expression of W525Acort ${ }^{\mathrm{r}}$ - 

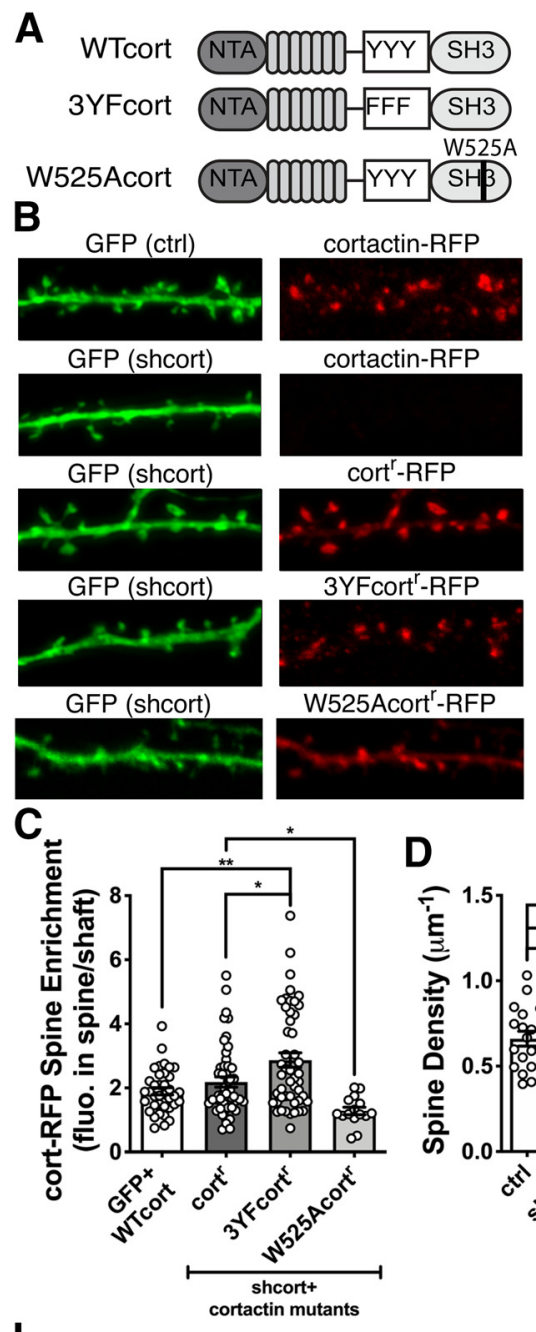

I

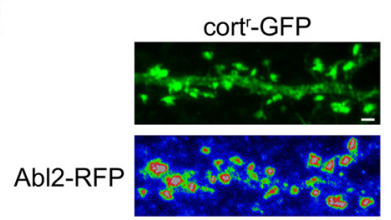

D

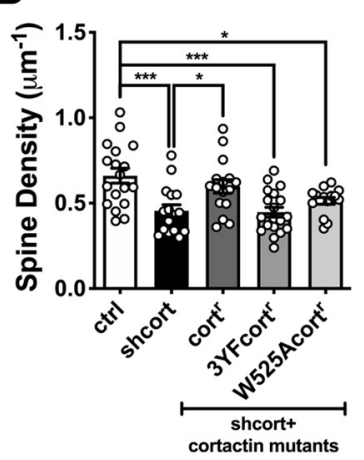

3YFcort'-GFP

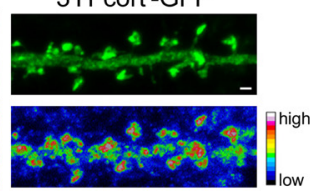

$\mathbf{E}$

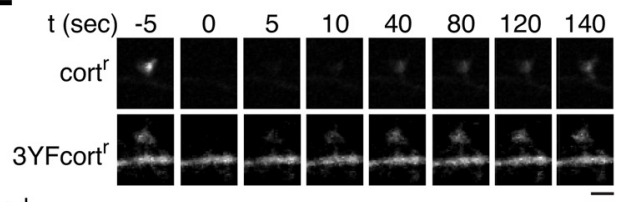
Merged
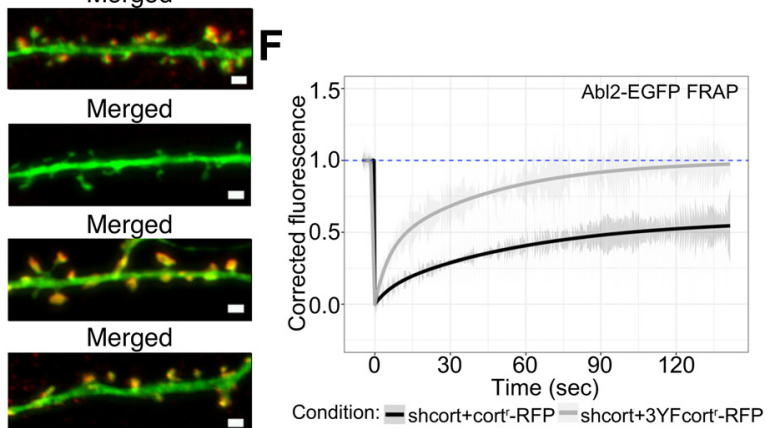

Merged

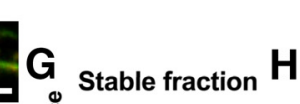

$H$
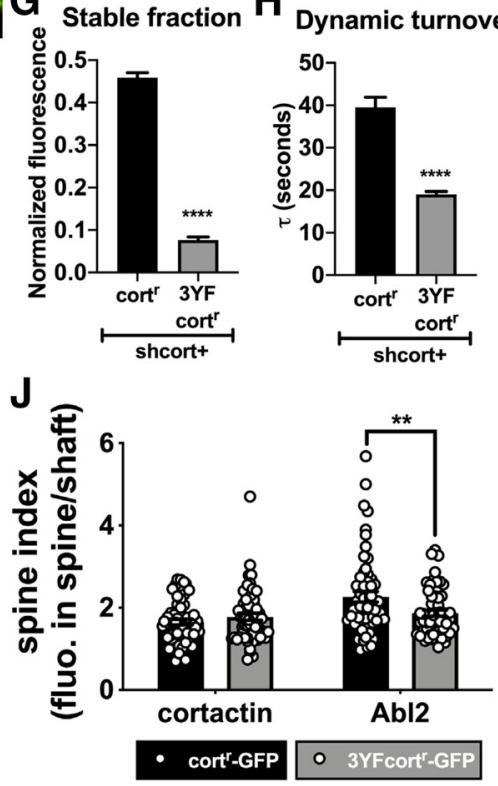

Figure 3. Cortactin phosphorylation is critical to support dendritic spine stability. $\boldsymbol{A}$, Illustration of cortactin domain mutants used in the experiment. $\boldsymbol{B}$, Representative confocal images of neurons transfected with GFP-tagged control vector or shcort. Cortactin KD neurons are cotransfected with cortactin-RFP, cort'-RFP, 3YFcort'-RFP, or W525Acort'-RFP. Scale bar, $1 \mu$ m. C, The spine enrichment of cortactin-RFP, cort'-RFP, 3YFcort'-RFP, or W525Acort'-RFP in control and cortactin KD neurons shows that cort'-RFP and $3 Y$ FFort'-RFP enrich in spines as well as cortactinRFP. W525Acort'-RFP exhibits weak spine enrichment (mean $=1.278$ ) but is significantly less than cort'-RFP. Data are mean \pm SEM $(n=15-48$ neurons/group). $* p<0.05 ; * * p<0.01 ;$ ordinary one-way ANOVA with Tukey multiple comparisons test. $\boldsymbol{D}$, Dendritic spine density of control neurons and cortactin KD neurons. Cortactin KD shows reduced spine density. Complementation with cort'-RFP, but not 3YFcort'-RFP or W525Acort'-RFP, restores spine density. Data are mean \pm SEM ( $n=15-19$ neurons/group). $* p<0.05 ; * * * p<0.001 ;$ ordinary one-way ANOVA with Tukey multiple comparisons test. $\boldsymbol{E}$, Representative time-lapse confocal images of Abl2-EGFP coexpressed with cort'-RFP, or 3YFcort'-RFP in cortactin KD neurons. Only the Abl2-EGFP channel is shown. Time $=0$ s indicates the frame immediately following photobleaching. Scale bar, $2 \mu \mathrm{m}$. $\boldsymbol{F}$, Abl2-EGFP turnover was fit to a one-phase exponential curve. In the presence of 3YFcort'-RFP, Abl2 has a lower stable fraction (G) and faster turnover kinetics $(\boldsymbol{H})$. Data are mean \pm SEM ( $n=11$ spines/group). $* * * * p<0.0001$ (unpaired $t$ test). $\boldsymbol{I}$, Representative confocal images show the spine enrichment of Abl2-RFP when coexpressed with cort'-GFP or 3YF-cort'-GFP. $J$, The spine enrichment of Abl2-RFP was significantly reduced when coexpressed with 3YF-cort'-GFP compared with the coexpression with cort'-GFP. Data are mean \pm SEM ( $n=57-59$ spines/group). $* * p<0.01$ (multiple $t$ test with Holm-Sidak method).

RFP, which did not enrich in spines, did not rescue this reduction in dendritic spine density (Fig. 3D). Interestingly, although it was more enriched in spines than $\operatorname{cort}^{\mathrm{r}}$-RFP, 3 YFcort ${ }^{\mathrm{r}}$-RFP did not rescue the reduction in dendritic spine density resulting from cortactin $\mathrm{KD}$ (Fig. $3 D$; summarized in Table 1). Phosphorylation of Y421 or Y466 is necessary for Abl2 binding to cortactin both in vitro and in cells, suggesting that this deficit in dendritic spine density could result from decreased Abl2:cortactin interaction in dendritic spines.

To test whether disrupted cortactin phosphorylation impaired spine stability by impacting Abl2 tethering in spines, we coexpressed Abl2-EGFP with cort $^{\mathrm{r}}$-RFP or 3 YFcort $^{\mathrm{r}}$-RFP in cortactin KD neurons and performed FRAP analysis on Abl2-EGFP (Fig. $3 E-H)$. When coexpressed with cort ${ }^{\mathrm{r}}$-RFP, a high proportion $(45.9 \pm 1.1 \%$; Fig. $3 F, G)$ of Abl2-EGFP does not recover, indicating stable tethering in the spine. The dynamic Abl2-EGFP pool exhibits slow, one-phase recovery $(\tau=39.5 \pm 2.4 \mathrm{~s}$; Fig. $3 F, H)$. However, coexpression of Abl2-EGFP with 3 YFcort ${ }^{\mathrm{r}}$-RFP resulted in a reduction of the stable pool of Abl2-EGFP ( $7.7 \pm 0.7 \%$; Fig. $3 F, G)$ and faster turnover of the dynamic proportion $(\tau=$ $19.0 \pm 0.7$ s; Fig. $3 F, H$ ), indicating reduced $\mathrm{Abl} 2$ tethering within spines. In addition, the spine enrichment of Abl2-RFP was 


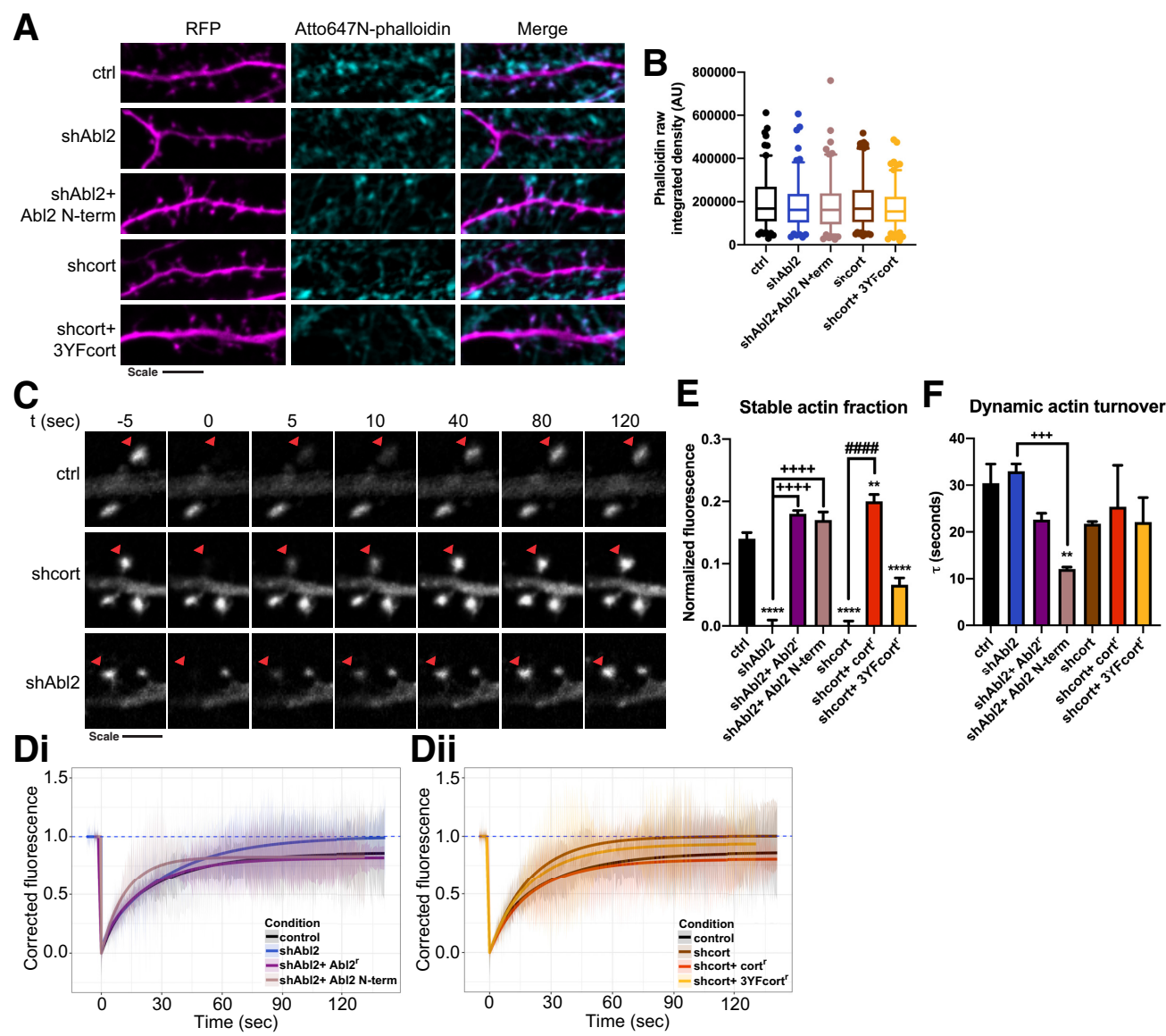

Figure 4. Abl2 and cortactin are required to maintain the stable actin pool in dendritic spines. $A$, Representative confocal images of neurons transfected with control vector, shAbl2, shAbl2 reconstituted with Abl2 mutants, shcort, or shcort reconstituted with cortactin mutants. Neurons were stained for RFP and with a fluorescent phalloidin to visualize actin filaments in spines. Scale bar, $5 \mu \mathrm{m}$. B, Quantified intensity of fluorescent phalloidin. Data are displayed as box (25th percentile, median, and 75th percentile) and whisker (5\%-95\%) plots ( $n=126-183$ spines/ condition). $p>0.999$ (Kruskal-Wallis test with Dunn's multiple comparison test). C, Representative time-lapse confocal images of GFP-actin expressed in dendritic spines of control neurons or neurons with Abl2 or cortactin KD. Time $=0$ s indicates the frame immediately following photobleaching. Red arrowhead indicates the spine that was photobleached. Scale bar, $2 \mu \mathrm{m}$. $\boldsymbol{D}, \mathrm{GFP}$ actin FRAP was fit to a two-phase exponential recovery curve. Recovery curves are shown for neurons transfected with control vector, shAbl2, and shAbl2 reconstituted with Abl2'-RFP or Abl2 mutants (Di) or shcort and shcort reconstituted with cortactin'-RFP or cortactin mutants (Dii). GFP-actin fluorescence does not recover fully in control neurons, but it reaches full recovery when Abl2 or cortactin is knocked down. The stable actin fraction $(\boldsymbol{E})$ and dynamic actin turnover $(\boldsymbol{F})$, computed from the fitted curve, are shown for each condition. Data are mean \pm SEM $(n=16-$ 28 spines/condition). $* * p<0.01 ;{ }^{++} p<0.001, * * * *,{ }^{++++, \# \#} p<0.0001$; ordinary one-way ANOVA with Tukey multiple comparisons test.

significantly reduced when coexpressed with 3 YFcort ${ }^{\mathrm{r}}$-GFP, compared with coexpression with cort $^{\mathrm{r}}$-GFP (Fig. 3I,J). These data, together with the findings that the kinase-inactive $\mathrm{Abl} 2$ and phospho-cortactin-binding defective Abl2-R198K mutant do not support normal spine density (Fig. 1D), strongly suggest that Abl2-mediated cortactin phosphorylation and subsequent Abl2 binding to phospho-cortactin promote $\mathrm{Abl} 2$ tethering within the spine, thereby promoting dendritic spine stability (summarized in Table 1).

\section{$\mathrm{Abl} 2$ and cortactin are required to maintain the stable actin pool in dendritic spines}

Cortactin-mediated enrichment of $\mathrm{Abl} 2$ into dendritic spines is critical to maintain normal dendritic spine density (Figs. 1D, $3 D$ ). However, this raised the central mechanistic question of whether and how the regulation of actin polymerization and stability by $\mathrm{Abl} 2$ and/or cortactin are important to promote normal spine density. Thus, we asked whether alterations in these proteins impacted actin levels and turnover and how these alterations relate to changes in net dendritic spine density, as well as spine size. First, we measured filamentous actin levels using Atto647N-phalloidin staining of fixed cells and quantified ROIs corresponding to dendritic spines, guided by expression of RFP that revealed neuronal structure (Fig. $4 A, B$ ). Although filamentous actin levels were broadly distributed among spines, we did not observe shifts in the distribution of total actin levels under any condition (Fig. 4B).

We also performed FRAP analyses on GFP-actin to test whether manipulation of $\mathrm{Abl} 2$ or cortactin impacted the relative proportions of dynamic versus stable actin content of the spine, and how this related to alterations in spine size or density (Fig. $4 C-F)$. Actin recovery time constants are best fit to a two phase recovery (Star et al., 2002; Koskinen et al., 2012): a fast component representing diffusion of GFP-actin monomers into the bleached spine $\left(\tau_{\text {monomer }}=\sim 1.5-3 \mathrm{~s}\right)$ and a slower component representing GFP-actin incorporation into the dynamic polymerizing actin network. The small unrecovered fraction represents stable actin that recovers on the order of 10 s of minutes.

Consistent with previous reports (Star et al., 2002; Koskinen et al., 2012; Koskinen and Hotulainen, 2014), we found that $13.9 \pm 1.0 \%$ of the GFP-actin fluorescence did not recover (stable fraction) after photobleaching (Fig. 4C-E). Abl2 or cortactin $\mathrm{KD}$ results in a complete recovery of GFP-actin fluorescence (Fig. $4 D, E)$, indicating, at the population level, that loss of Abl2 and 
A

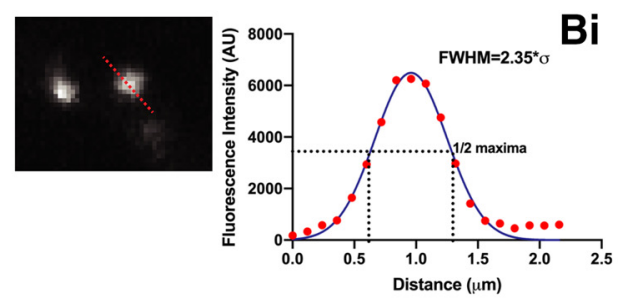

Bi

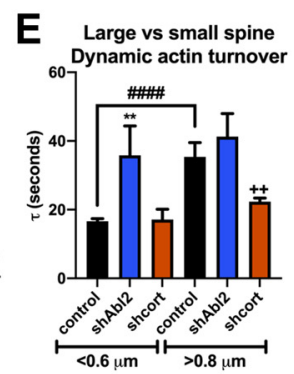

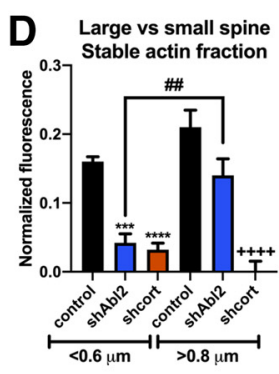

$\mathbf{F}$

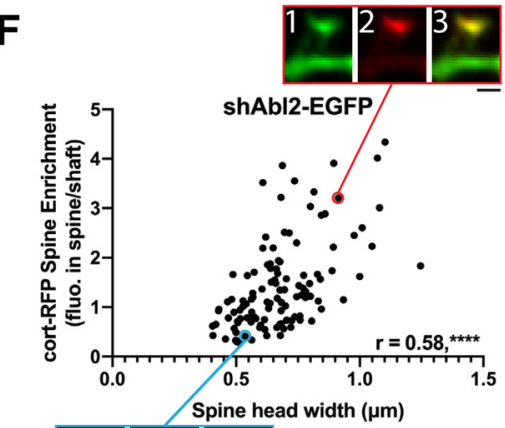

G
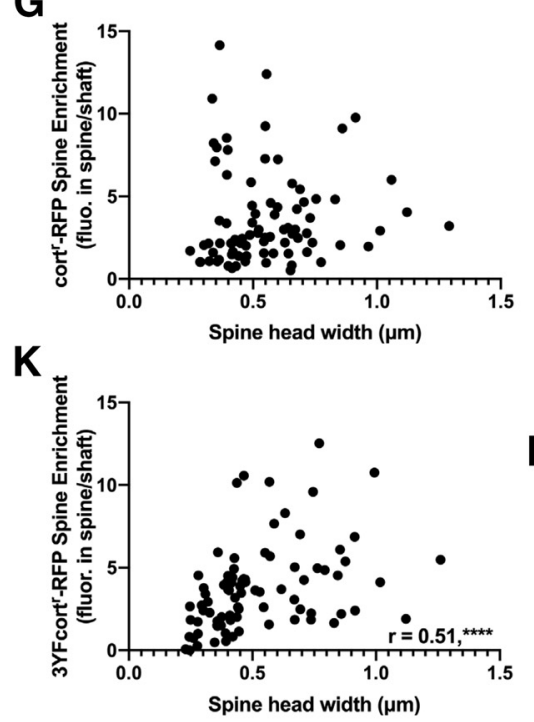

Bii
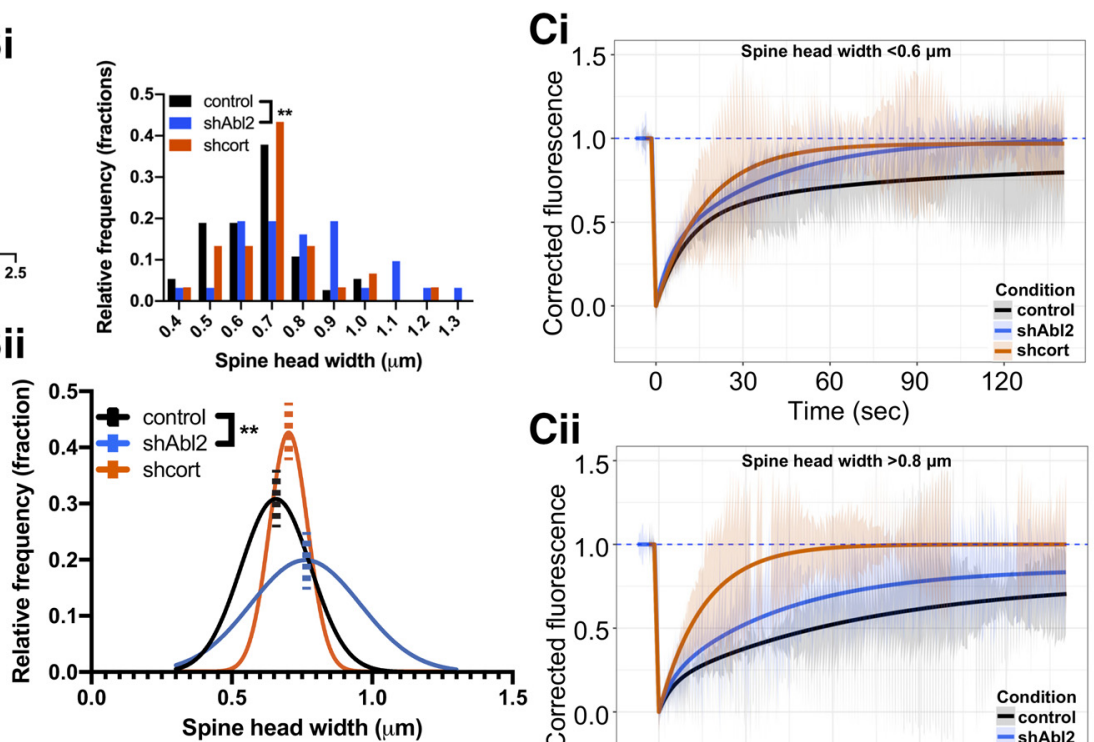

H
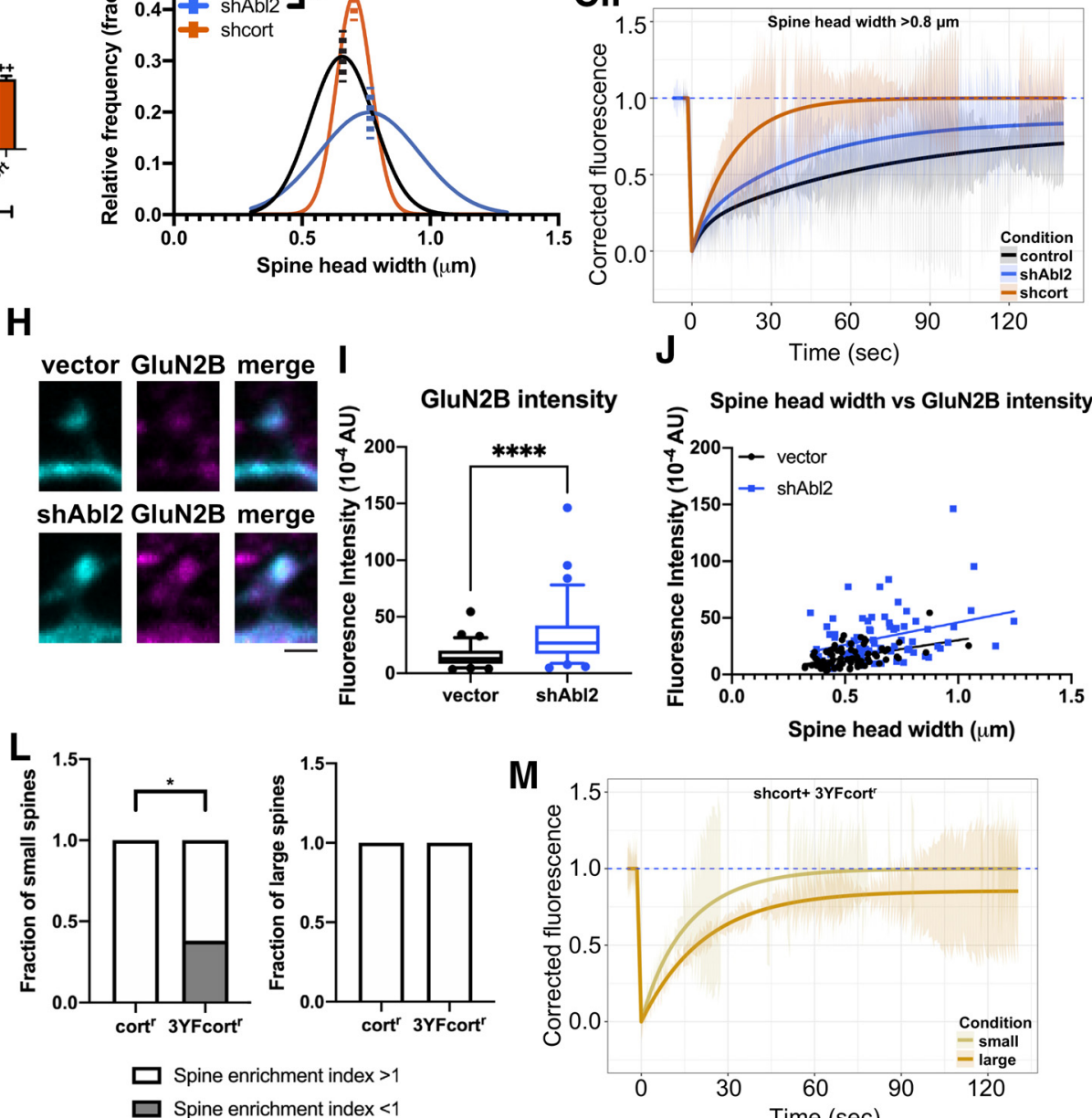

M

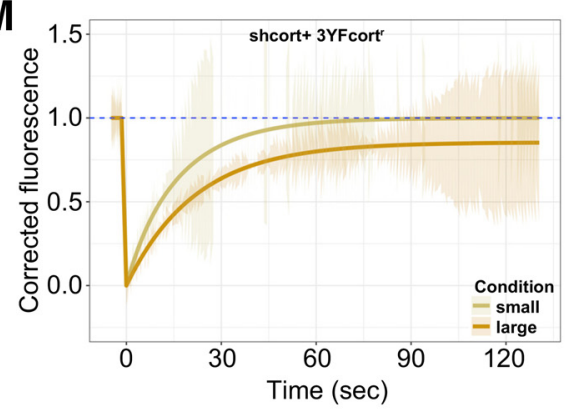

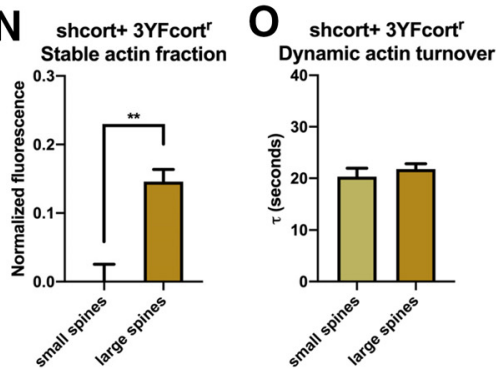

Figure 5. Loss of Abl2 and cortactin differentially impacts small versus large dendritic spines. $\boldsymbol{A}$, FWHM was measured for each spine head subjected to photobleaching by plotting the fluorescence profile across a line and fitting to a Gaussian curve. $\boldsymbol{B}$, The distribution of the average spine head width of each photobleached spine is shown both as a frequency distribution (Bi) and fit to a Gaussian curve (Bii). Dotted line indicates middle of the curves. The shAbl2 spine heads subjected to photobleaching are significantly enlarged compared with control spines (control, $n=26$; shAbl2, $n=24$ spines). $* * p<0.01$ (Welch's $t$ test). To account for any differences in populations of spine heads that were photobleached, data were parsed into small spines $<0.6 \mu \mathrm{m}(\boldsymbol{C})$ and large spines $>0.8 \mu \mathrm{m}$ (Cii) for control, shAbl2, and shcort data, and recovery curves were generated. Difference in the stable actin fraction (D) and dynamic actin turnover $(\boldsymbol{E})$ is shown. Data are mean \pm SEM ( $n=6-13$ spines/condition). $* *, \#,++p<0.01, * * * p<0.001, * * * *, \# \#,++++p<0.0001$ ordinary one-way ANOVA with Tukey multiple comparisons test. $\boldsymbol{F}$, Scatterplot for spine head width versus cort-RFP spine enrichment in AbI2 KD neurons shows a positive correlation ( $n=117$ spines). $r=0.58$ (Pearson correlation test). $* * * * p<0.0001$. Representative confocal images are shown for a small spine (bottom), demarked with a blue outline, and a large spine (top), demarked with a red outline. Channels: (1) shAbl2-EGFP, (2) cort-RFP, and (3) merged. Scale bar, $1 \mu \mathrm{m}$. G, Scatterplot for spine head width and cort'-RFP spine enrichment in cortactin KD neurons shows no correlation ( $n=83$ spines). 
cortactin yields spines that lack a stable actin pool. Reexpression of Abl2 ${ }^{\mathrm{r}}$-RFP or cort $^{\mathrm{r}}$-RFP restored the pool of stable actin when expressed in $\mathrm{Abl} 2$ or cortactin KD neurons, respectively (Fig. 4D, $E)$. Interestingly, complementation of $\mathrm{Abl} 2 \mathrm{KD}$ neurons with Abl2 N-term-RFP, which rescued dendritic spine loss and cortactin enrichment in spines, also completely rescued the stable actin pool (Fig. 4Di,E). Unexpectedly, rescue with the Abl2 Nterm-RFP also increased the recovery rate of the dynamic pool $\left(\tau_{\text {control }}=30.5 \pm 4.1 \mathrm{~s}, \tau_{\text {Abl2 }} \mathrm{N}\right.$-term $=12.1 \pm 0.4 \mathrm{~s}$; Fig. $\left.4 D i, F\right)$. This may result from the elevated expression levels and consequently elevated kinase activity of $\mathrm{Abl} 2 \mathrm{~N}$-term compared with full-length Abl2 (Peacock et al., 2007).

We next tested how disruption of cortactin phosphorylation impacts GFP-actin turnover. Complementation of cortactin KD neurons with $3 \mathrm{YFcort}^{\mathrm{r}}$-RFP yielded partial rescue of the stable actin pool (stable fraction $=13.9 \pm 1.0 \%$ for control and $6.6 \pm 1.1 \%$ for 3 YFcort $^{\mathrm{r}}$-RFP; Fig. 4Dii,E). 3 YFcort $^{\mathrm{r}}$-RFP did not fully rescue dendritic spine density (Fig. $3 D$ ), indicating that the remaining stable actin pool is not sufficient to stabilize spines. These data demonstrate that $\mathrm{Abl} 2$ and cortactin are novel key regulators of the stable actin pool in dendritic spines.

\section{Loss of Abl2 and cortactin differentially impacts small versus large dendritic spines}

Previous measurements of actin turnover in dendritic spines show that the amount of stable actin is proportional to the spine volume squared (Honkura et al., 2008). A subset of remaining spines following Abl2 depletion exhibited gradual spine head size enlargement (Y. C. Lin et al., 2013; Xiao et al., 2016). These findings led us to examine how changes in actin pool sizes and recovery kinetics correlated with spine head size. For each spine analyzed, we measured the FWHM of the spine head in the prebleach frames and took the average of those frames to approximate individual spine head widths (Fig. 5A). The distribution of spine head widths of photobleached spines was significantly broader in the Abl2 KD condition than in control spines (Fig. $5 B$ ), consistent with measurements made previously (Y. C. Lin et al., 2013). In notable contrast, the distribution of spine head widths for cortactin $\mathrm{KD}$ photobleached spines was not

$\leftarrow$

$r=0.19$ (Pearson correlation test). $p=0.078$. $\boldsymbol{H}$, Representative confocal images of control and AbI2 KD neurons stained for endogenous GluN2B (magenta) and a EGFP fill (cyan). I, GluN2B fluorescence intensity was quantified by selecting an ROI over the spine head, guided by the EGFP channel. Data are displayed as box (25th percentile, median, and 75th percentile) and whisker (5\%-95\%) plots ( $n=77$ or 78 spines/condition). $* * * * p<0.0001$ (Mann-Whitney test). J, Scatterplot for spine head width and GluN2B spine fluorescence intensity in control and Abl2 KD neurons shows a positive correlation ( $n=77$ or 78 spines/condition). Vector: $r=0.46, p<0.0001$; shAbl2: $r=0.32, p<0.01$ (Pearson correlation test). Linear regression modeling shows no significant difference between slopes $(p=0.64)$, but different $y$ intercepts $(p<0.0001)$ for the control and Abl2 KD datasets (extra sum-ofsquares $F$ test). $\boldsymbol{K}$, Scatterplot for spine head width and 3YFcort'-RFP spine enrichment in cortactin KD neurons shows a positive correlation ( $n=83$ spines). $* * * * p<0.0001 . r=0.51$ (Pearson correlation test). $L$, The fraction of small and large spines that show preferential spine enrichment (spine enrichment $>1$ ) from the population of cort'-RFP and 3YFcort'-RFP spines. A significant fraction of small 3YFcort'-RFP spines do not have a preferential enrichment in spines (spine enrichment $<1$ ) compared with small cort'-RFP spines (small: $n=10$

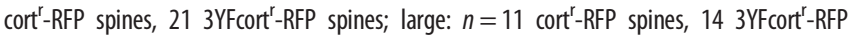
spines). $* p<0.05$ (Fisher's exact test). $\boldsymbol{M}$, Because of a significant correlation between spine head width and 3YFcort'-RFP spine enrichment, and differences in enrichment between small and large spines, data for GFP-actin FRAP in shcort +3 YFcort'-RFP neurons were parsed into small spines $<0.6 \mu \mathrm{m}$ and large spines $>0.8 \mu \mathrm{m}$, and recovery curves were generated. Difference in the stable actin fraction $(\boldsymbol{N})$ is shown with no changes in the dynamic actin turnover (0). Data are mean \pm SEM ( $n=5$ spines/condition). $* * p<0.01$ ( $t$ test). significantly different from control spines (Fig. 5B). These differences in the populations of spine head widths raise the question of whether spines of different head sizes exhibit differences in the stable pool in Abl2- or cortactin-KD neurons.

To test how the relative size of the stable actin pool related to spine size, we segmented our FRAP dataset based on spine head widths and classified small spines based on FWHM $<0.6 \mu \mathrm{m}$ and large spines with FWHM $>0.8 \mu \mathrm{m},<1$ and $>1$ SD from the control mean, respectively. Small and large spines in the control and cortactin KD datasets represent $\sim 25 \%$ of the total population of spines analyzed in Figure 4. However, for the Abl2 KD dataset, small spines account for $\sim 20 \%$ of the total population, while large spines are $40 \%$. Small Abl2 KD spines, as well as both small and large cortactin $\mathrm{KD}$ spines, exhibited significantly smaller stable actin pools compared with their respective control (Fig. 5C,D), consistent with the pooled data in Figure $4 D, E$. Dynamic actin turnover rates were not significantly changed in any of these conditions (Fig. 5E). The stable pool size trended toward an increase in larger spine head widths, consistent with a previous report (Honkura et al., 2008) (Fig. 5Cii,D), although we could not establish significance in our dataset because of the small number of large spines in the control neuron comparison group. Large Abl2 KD spines had a significantly larger stable actin pool than small $\mathrm{Abl} 2 \mathrm{KD}$ spines, and this was not significantly different from the stable actin pool size in large control spines (Fig. 5Cii,D). Together, these data suggest that, while cortactin is absolutely required to maintain the stable actin pool in all spines, the subset of large spines in Abl2-deficient neurons retain the stable actin pool.

The remaining stable actin pool in large Abl2 $\mathrm{KD}$ spines suggested that there may be an Abl2-independent enrichment of cortactin in these large spines, that enables actin stabilization. To determine whether cortactin enrichment in Abl2 KD spines correlates with spine head size, we quantified the FWHM and cortRFP spine enrichment for spines on Abl2 KD neurons (Fig. $5 F$ ). We found a positive Pearson correlation $(r=0.58)$ between spine size and cortactin enrichment. In cortactin KD neurons reconstituted with $\operatorname{cort}^{\mathrm{r}}$-RFP, there is no significant correlation between spine head width and cortactin spine enrichment (Fig. 5G). This suggests that elevated cortactin levels in large Abl2 KD spines are sufficient to support a stable actin pool and protect these spines from destabilization.

In addition to structural aberrations, $\mathrm{Abl} 2 \mathrm{KO}$ neurons have been shown to have increased NMDAR currents mediated by GluN2B subunit containing NMDARs (Xiao et al., 2016). We stained Abl2 KD neurons with an antibody that binds an extracellular epitope on GluN2B subunit of the NMDAR receptor (Fig. $5 \mathrm{H}-J)$, to test whether enlarged $\mathrm{Abl} 2 \mathrm{KD}$ spines, which retain cortactin and a stable actin pool, have more surface GluN2B. ROIs encompassing spines in $\mathrm{Abl} 2 \mathrm{KD}$ neurons contain more GluN2B than control neurons (Fig. 5H,I). In both control and $\mathrm{Abl} 2 \mathrm{KD}$-transfected neurons, there was a strong positive correlation between spine head size and GluN2B intensity ( 0.46 and 0.32 , respectively; Fig. 5J). Using linear regression modeling and extra sum-of-squares $F$ test, we found the relationship had the same slope, but two very different $y$ intercepts, indicating a global increase in spine GluN2B levels in Abl2 KD neurons.

The 3YFcort mutant is enriched in spines to a greater extent than WT cort (Fig. 3C), but it untethers Abl2 from spines (Fig. $3 \mathrm{~F}-\mathrm{H}$ ) and only partially rescues the stable actin pool (Fig. 4Dii, $E)$, resulting in a lower spine density (Fig. $3 D$ ). Therefore, we used the parsing method above to document and quantify the Abl2-independent enrichment of cortactin, using 3 YFcort ${ }^{\mathrm{r}}$-RFP, 


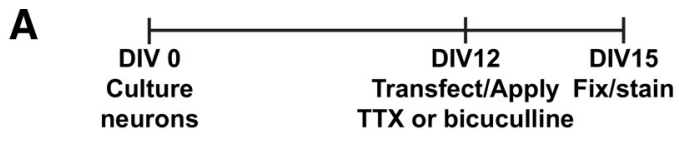

B

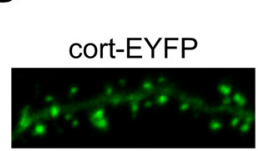

Untreated

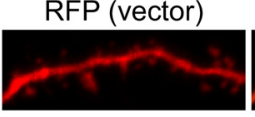

cort-EYFP

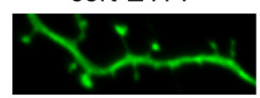

RFP (shAbl2)

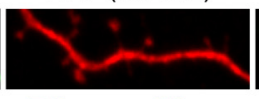

Bicuculline

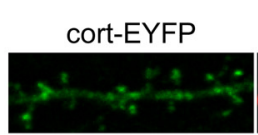

cort-EYFP

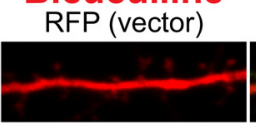

RFP (shAbl2)

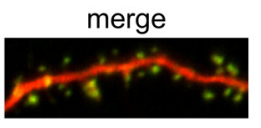

merge

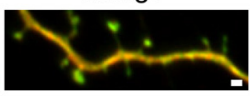

merge

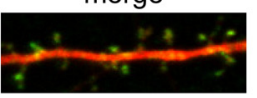

merge

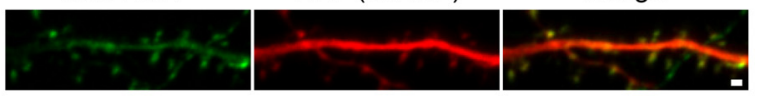

TTX

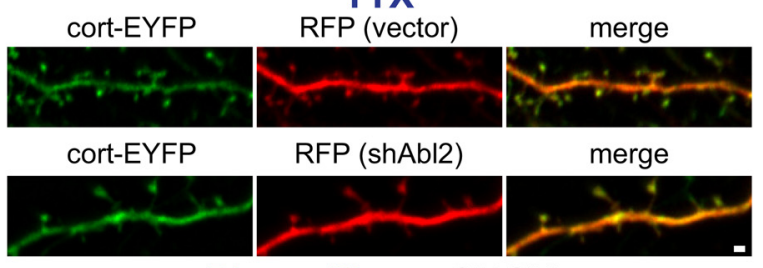

Bicuculline + CNQX
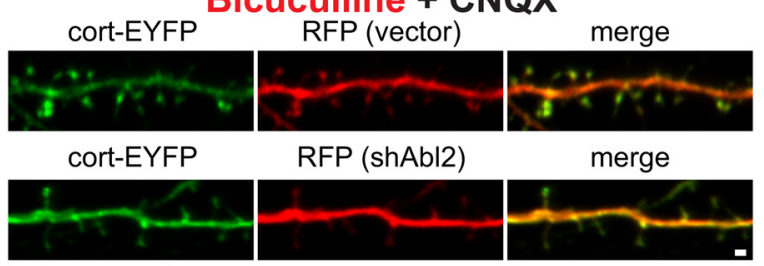

merge

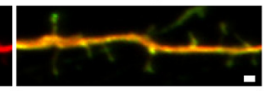

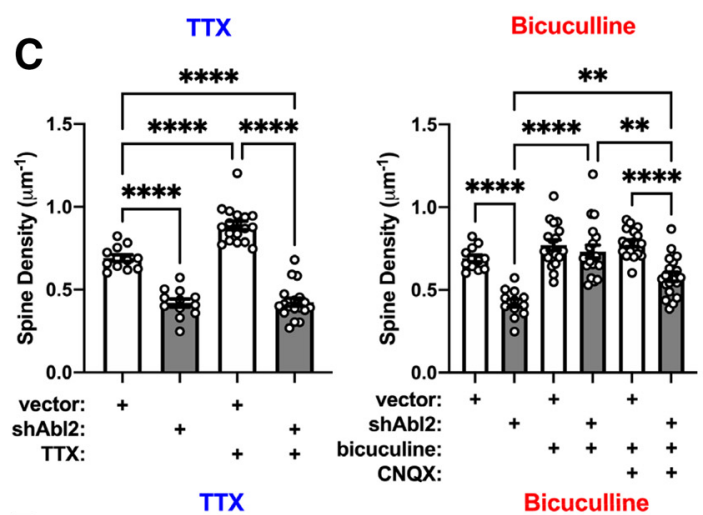

E TTX

Bicuculline
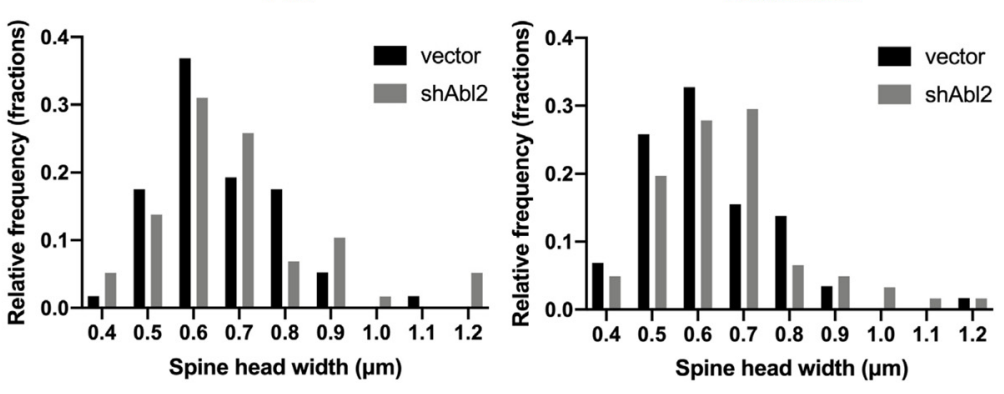

$\mathbf{F}$
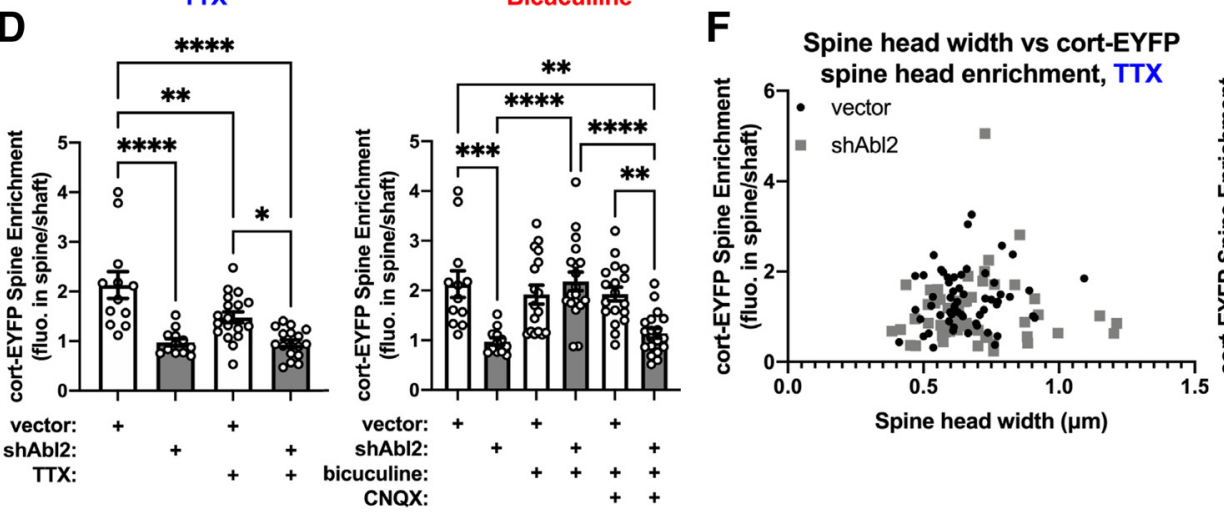

Spine head width vs cort-EYFP spine head enrichment, Bicuculline

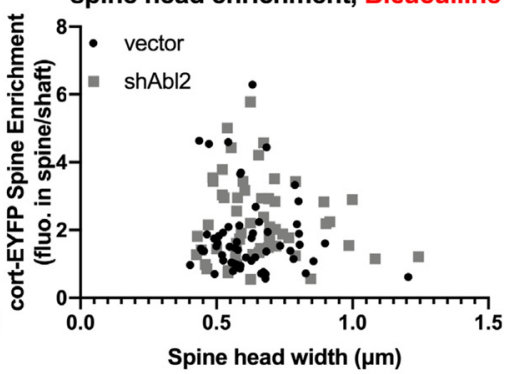

Figure 6. Tonic enhancement of synaptic activity rescues cortactin depletion and spine loss in Abl2 KD neurons. A, Experimental setup. Neurons were cultured for $12 \mathrm{~d}$ before knocking down

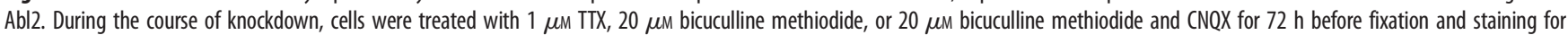
YFP and RFP, to visualize cortactin localization and cell morphology, respectively. $\boldsymbol{B}$, Representative confocal images of neurons cotransfected with control vector or shAbl2 and cortactin-EYFP. Scale bar, $1 \mu \mathrm{m}$. C, Dendritic spine density of control and Abl2 KD neurons treated with TTX, bicuculline, or bicuculline and CNQX. TTX-treated neurons show a reduced dendritic spine density upon $\mathrm{Abl} 2 \mathrm{KD}$, similar to basal conditions. However, treatment of neurons with bicuculline rescues dendritic spine deficits upon Abl2 KD. This rescue can be reversed on Abl2 KD neurons by simultaneously treating neurons with bicuculline and CNQX. Data are mean \pm SEM ( $n=12-19$ neurons/condition). $* * p<0.01 ; * * * * p<0.0001$; ordinary one-way ANOVA with Tukey multiple comparisons test. $\boldsymbol{D}$, Spine enrichment of cortactin-EYFP in control and Abl2 KD neurons treated with TTX and bicuculline. Cortactin enrichment in spines is retained in AbL2 KD neurons treated with bicuculline. Data are mean \pm SEM $(n=12-19$ neurons/condition). $* p<0.05 ; * * p<0.01 ; * * * p<0.001 ; * * * * p<0.0001$; ordinary one-way ANOVA with Tukey multiple comparisons test. $\boldsymbol{E}$, The distribution of spine head widths in control and Abl2 KD conditions treated with TTX and bicuculline is shown as a frequency distribution. Abl2 KD spines do not show an enlargement compared with control spines when treated with TTX or bicuculline ( $n=57-61$ spines/condition). TTX, vector versus shAbl2: $p=0.7434$; bicuculline, vector versus shAbl2: $p=0.2269$ (Mann-Whitney test). $\boldsymbol{F}$, Scatterplot for spine head width and cort-EYFP spine enrichment in control and AbI2 KD neurons treated with TTX and bicuculline does not show a positive correlation for any of the conditions ( $n=57-61$ spines/condition).

and how this correlated with actin stability in large versus small spines of Abl2 KD neurons. Similar to cortactin spine enrichment in Abl2 KD neurons, 3YFcort ${ }^{\mathrm{r}}$-RFP spine enrichment is positively correlated with spine head size (Pearson correlation $r=0.51$ ) (Fig. 5K). Furthermore, a significant proportion of small spines do not show spine enrichment of 3YFcort ${ }^{\mathrm{r}}$-RFP (Fig. 5L). Given differential enrichment of 3YFcort in small versus large spines, we performed GFP-actin FRAP in these small and large 
spines (Fig. 5M). While in aggregate, the 3 YFcort $^{\mathrm{r}}$-RFP data had a partial rescue of the stable actin pool (Fig. 4Dii,E), we found that small spines had no stable actin pool, similar to cort $\mathrm{KD}$ neurons, and large spines had stable actin pools comparable in size to spines in control neurons (stable fraction $=14.6 \pm 1.8 \%$; Fig. $5 N$ ). No significant changes in the dynamic actin turnover were observed in the cort $\mathrm{KD}+3 \mathrm{YFcort}^{\mathrm{r}}$-RFP spines regardless of size (Fig. 5O). These data indicate that, even while cortactin tethering of Abl2 is important for maintaining small dendritic spines, in the absence of Abl2, cortactin associates with another determinant in the spine that scales with spine size.

\section{Tonic enhancement of synaptic activity rescues cortactin depletion and spine loss in Abl2 KD neurons}

Synaptic activity bidirectionally reorganizes the molecular composition and structure of dendritic spines. Actin is a major substrate for bidirectional plasticity, as changes in actin dynamics rapidly correlate with changes in dendritic spine structure (Fukazawa et al., 2003; Okamoto et al., 2004). Moreover, manipulations of activity modulate the translocation of cortactin and other synaptic proteins into and out of the spine (Hering and Sheng, 2003; Iki et al., 2005; Bosch et al., 2014; K. Kim et al., 2015). Given the impact of Abl2 and cortactin on the stable actin pool (Figs. 4, 5) and this unique link between spine head size and cortactin spine enrichment in Abl2-deficient neurons (Fig. 5F,K-O), we asked whether tonic changes in activity impacted these phenotypes.

During the duration of Abl2 KD, we treated cultured neurons with either $1 \mu \mathrm{M}$ TTX or $20 \mu \mathrm{m}$ bicuculline to ask how chronic blockade or enhancement of activity, respectively, modulated dendritic spine density, cortactin localization, and gross changes in dendritic spine structure (Fig. 6A). Compared with untreated neurons, TTX-treated neurons expressing the control vector had a significant increase in spine density, while bicuculline treatment did not significantly impact spine density (Fig. 6C). TTX did not impact Abl2-deficient neurons compared with control transfected cells, they had a significantly reduced dendritic spine density (Fig. $6 B, C$ ) and a $31 \pm 10 \%$ reduced spine enrichment of cortactin, similar to untreated Abl2-deficient neurons (Fig. 6B,D). Interestingly, tonically increasing activity with bicuculline rescued the reduced spine density in $\mathrm{Abl} 2 \mathrm{KD}$ neurons (Fig. 6B,C) and maintained cortactin enrichment in these spines (Fig. 6B,D). This rescue effect of bicuculline on $\mathrm{Abl} 2 \mathrm{KD}$ spine density and cortactin spine enrichment was blocked by simultaneously applying $20 \mu \mathrm{M}$ bicuculline and $20 \mu \mathrm{M}$ CNQX (Fig. $6 B-D$ ), further supporting a role for neuronal activity in cortactin regulation.

Spine head width distribution of Abl2 KD neurons was normalized in bicuculline-treated neurons to mirror the head widths observed in control neurons: the distribution of spine head widths was no longer right-shifted (Fig. $6 E$, right), reflecting that loss of Abl2 no longer results in a significant enlargement of spine heads. These data indicate that tonically increasing activity compensates for Abl2 deficiency. TTX did not impact net cortactin depletion from spines or net spine loss in Abl2 KD neurons, but it normalized spine head size (Fig. $6 E$, left). Consistent with normalization of dendritic spine head widths in Abl2 KD neurons by both TTX and bicuculline, we no longer observed the correlation between spine head width and cortactin spine enrichment (Fig. 6F) observed in $\mathrm{Abl} 2 \mathrm{KD}$ neurons in basal conditions (Fig. $5 F$ ).

\section{Discussion}

Reductions in dendritic spine density are associated with cognitive, perceptual, and affective deficits in psychiatric, neurodevelopmental, and neurodegenerative disorders. The actin cytoskeleton, consisting of both dynamic and stable pools, is a major determinant of spine structure and stability. Here, we identify Abl2 and cortactin as key regulators of the stable actin pool in dendritic spines. We show that disruption of $\mathrm{Abl} 2 \mathrm{SH} 2$ domain binding to phosphorylated cortactin reduces enrichment of both proteins in spines and disrupts the stable actin pool. Loss of the stable actin pool is associated with significant reductions in spine density. Curiously, we also find that a small subset of larger spines in Abl2-deficient neurons exhibit increased cortactin recruitment and a stable actin pool, suggesting that cortactin acts via both Abl2-dependent and independent mechanisms. Together, our results define new key regulators of the stable actin pool in spines and demonstrate the importance of stable actin in regulating spine shape and stability.

\section{A role for stable actin in dendritic spine stability}

Consistent with previous measurements, we find that only a small fraction of spine actin is stable (Star et al., 2002; Fukazawa et al., 2003; Honkura et al., 2008; Koskinen et al., 2014). This stable actin pool appears to localize to the spine base (Fukazawa et al., 2003; Bosch et al., 2014), where cortactin (Racz and Weinberg, 2004) and other actin binding proteins (Racz and Weinberg, 2006, 2008; Bosch et al., 2014) have also been shown to concentrate. We demonstrate that depletion of the stable actin pool is tightly associated with decreased dendritic spine stability and overall decreased spine density. Although stable actin is not very abundant in dendritic spines, it seems to play a major role in dendritic spine stability, raising the fundamental question of how it contributes to spine homeostasis.

The ongoing maintenance of spine shape and synaptic transmission requires continual dynamic actin polymerization and turnover (C. H. Kim and Lisman, 1999; Krucker et al., 2000; Honkura et al., 2008; K. Kim et al., 2015). It is possible that these dynamic actin filaments use the stable actin pool as a base for their nucleation. In maturing synapses, this dynamic pool also undergoes continual retrograde flow (Koskinen et al., 2014), which likely exerts force on the stable pool at the spine base. Here, the stable pool may play a supporting role to resist this force and stabilize the spine. The size of the stable pool scales with spine size (Honkura et al., 2008; Koskinen et al., 2012), consistent with possible roles as a support structure for the peripheral dynamic pool. Bosch et al., (2014) proposed that, during structural LTP, this stable actin network is transiently unbound by actin stabilizing proteins, becoming susceptible to severing and nucleation proteins that function to remodel the actin cytoskeleton (Bosch et al., 2014). Because of the limited number of proteins that were screened in this paper, the dynamics of Abl2 and cortactin were not measured in their study, but we postulate they would play major roles in intermediate phases of structural LTP.

Large bundles of actin filaments at the base of the spine are found enmeshed with the spine apparatus in a subset of spines (Capani et al., 2001). Although the function of the spine apparatus remains enigmatic, dendritic spines containing a spine apparatus are larger and have more potent synapses (Holbro et al., 2009). Furthermore, only spines that contain a spine apparatus grow during cLTP (Deller et al., 2003; Borczyk et al., 2019). Therefore, the stable actin pool may support the spine apparatus to promote spine stability. Previous work from Mikhaylova et al. (2018) demonstrated that caldendrin depletion also results in a loss of the stable actin pool and spine plasticity deficits. Furthermore, caldendrin depletion enhances cortactin turnover 

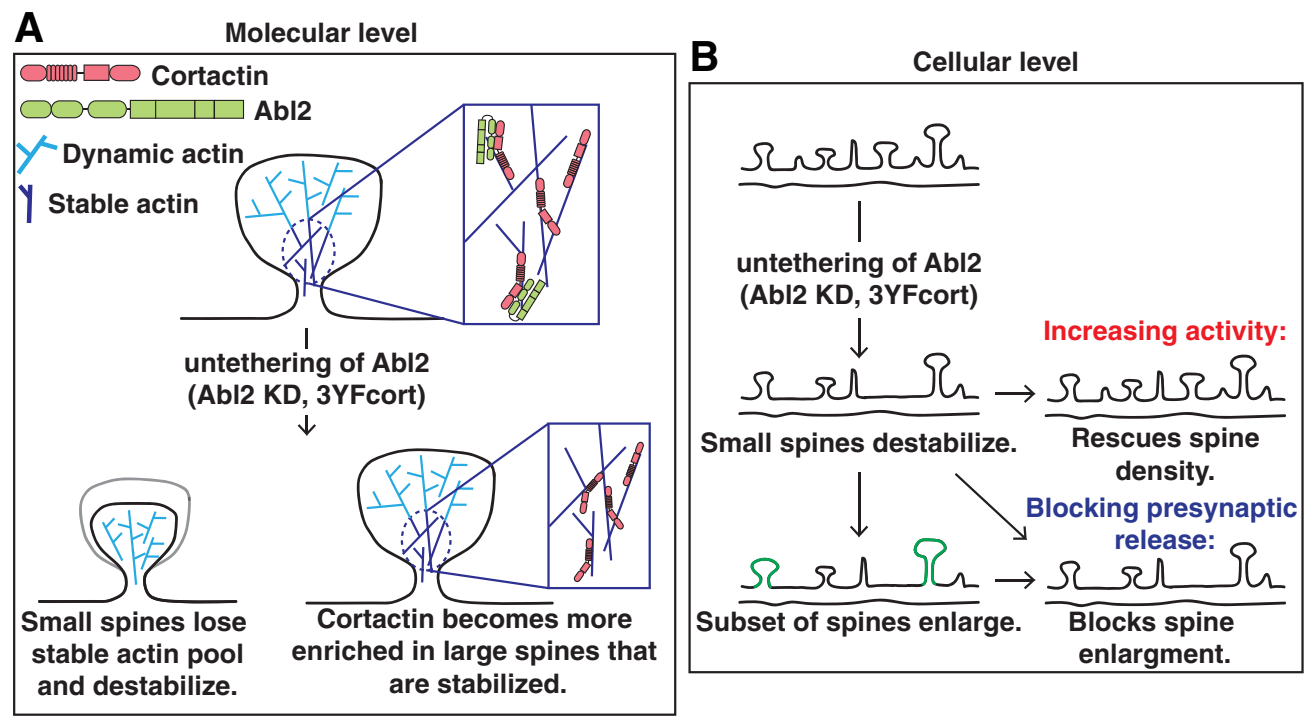

Figure 7. Model of Abl2:cortactin regulation of dendritic spine stability. A, At the molecular level, a subset of dendritic spine actin is stable (dark blue), whereas the majority is highly dynamic (light blue). The Abl2 SH2:phospho-cortactin interaction is essential in maintaining this pool of stable actin (top, inset). Dendritic spine loss following Abl2 KD is accompanied by loss of the stable actin pool in these small spines (bottom, left) and the redistribution of cortactin to large spines that retain a significant stable actin pool (bottom, right), by an Abl2-independent mechanism. $B, A$ cellular perspective of how Abl2 and cortactin impact dendritic spines. At homeostasis, dendritic spines are all different sizes and maintain different neurotransmitter and synaptic machinery. Loss of Abl2 results in the gradual loss of cortactin from small spines and their eventual destabilization. Spine loss can be prevented in Abl2 KD neurons by tonically increasing activity. Furthermore, spine loss is accompanied by the enlargement of some remaining spines, which can be prevented by blocking presynaptic release.

in dendritic spines, suggesting that these changes in spine actin stability may be the indirect result of cortactin loss from spines.

\section{How do Abl2 and cortactin maintain the stable pool of actin in spines?}

We show here that $\mathrm{Abl} 2$ and cortactin are essential to maintain the stable actin pool in dendritic spines. Although Abl2 and cortactin can each independently bind and stabilize actin filaments, they synergize at much lower binding stoichiometries in vitro (Courtemanche et al., 2015; Scherer et al., 2018). The Abl2 N-terminal half, which does not bind actin and only has weak effects on cortactin binding to actin, is sufficient to support the stable actin pool in Abl2-deficient neurons. The Abl2 kinase domain, located in the protein's N-terminus, phosphorylates cortactin, creating a binding site for the $\mathrm{Abl} 2 \mathrm{SH} 2$ domain. Mutation of this interface, either via point mutations to the $\mathrm{Abl} 2 \mathrm{SH} 2$ domain or the cortactin phosphorylation sites, reduces the stable actin pool and leads to spine loss. We hypothesize that Abl2 phosphorylation of, and tethering to, cortactin keeps cortactin in the spine, promoting interactions with actin and possibly other proteins that are critical for it to maintain the stable actin pool. We also find that the nonphosphorylatable (3YF) cortactin is more enriched in spines, and $\mathrm{Abl} 2$ is more dynamic in spines reconstituted with 3YFcort. Uncoupling from Abl2 may free up 3YFcort to interact with other binding partners in the spine, including, but not limited to, the Shank family of proteins (MacGillavry et al., 2016). In vitro, cortactin phosphorylation does not impact its affinity for actin (MacGrath and Koleske, 2012b) or ability to stabilize actin filaments (Courtemanche et al., 2015), suggesting that 3YFcort maintains the stable actin pool in a minor population of spines in the absence of Abl2 (Fig. 5K). However, Abl2: cortactin interactions stabilize cortactin binding to actin filaments (MacGrath and Koleske, 2012b), indicating that binding to Abl2 can act as a recruiting mechanism for cortactin to a subpopulation of filaments.

We also found that rescue of $\mathrm{Abl} 2$ with the $\mathrm{Abl} 2 \mathrm{~N}$-terminus yields much faster dynamic actin recovery. The Abl2 N-terminus expresses at elevated levels over Abl2, yielding increased Abl2 kinase activity (Peacock et al., 2010). In addition to cortactin, Abl family kinases phosphorylate N-WASp and WAVE2 (Stuart et al., 2006; M. M. Miller et al., 2010), which can both activate the Arp2/3 complex (Martinez-Quiles et al., 2004). Abl2 also phosphorylates the RhoA inhibitor p190RhoGAP (Sfakianos et al., 2007), which attenuates RhoA/ROCK signaling and could activate actin severing by cofilin (Bravo-Cordero et al., 2011). Increasing Arp2/3-mediated actin branching and cofilin severing would both result in increases in dynamic turnover.

How do large Abl2-deficient spines retain a stable actin pool? Depletion of Abl2 results in a significant loss of cortactin from spines and overall spine loss (Y. C. Lin et al., 2013; Xiao et al., 2016). Curiously, while net spine stability is decreased in Abl2deficient neurons, a subset of the remaining spines enlarge (Fig. 5). These large spines retain a stable actin pool and are enriched for cortactin. Previous work has also shown that the enlarged subset of spines in Abl2-deficient mice have larger GluN2Bmediated NMDAR currents (Xiao et al., 2016). We find that tonically increasing synaptic activity with bicuculline rescues cortactin spine enrichment in $\mathrm{Abl} 2 \mathrm{KD}$ neurons and overall dendritic spine stability (Fig. 7), and blocking presynaptic release with TTX prevents spine head enlargement. Paradoxically, other studies have shown NMDAR stimulation causes cortactin to redistribute from spines to dendrite shafts (Hering and Sheng, 2003; Iki et al., 2005), although it is unclear which class of NMDARs trigger cortactin exit from spines. It is clear that, in the absence of Abl2, some large spines retain the ability to maintain cortactin levels and a stable actin pool, suggesting that cortactin may respond differently to NMDARs, possibly dependent on their subunit composition. In addition to Abl2, cortactin interacts via its $\mathrm{SH} 3$ domain with the synaptic scaffolds SHANK2 (MacGillavry et al., 2016) and cortactin-binding protein 2 (Chen and Hsueh, 2012), suggesting that these large spines may tether cortactin by either of these proteins. Also, the cortactin repeats region of cortactin, which binds and stabilizes 
actin filaments (Weed et al., 2000; Scherer et al., 2018), is sufficient to localize cortactin in spines (Hering and Sheng, 2003), suggesting that interactions with actin may be sufficient. Neurotrophic cues, such as BNDF, have been proposed to drive cortactin into spines (Iki et al., 2005), where it would be able to interact with actin filaments. Therefore, retaining cortactin localization in spines via tethering to various synaptic scaffolds or even actin itself likely compensates for a loss of Abl2 and its disruption of the stable actin pool.

\section{A model for Abl2 and cortactin in regulation of spine stable actin}

While dendritic spines contain two kinetically distinct pools of spine actin, the function of the stable actin pool has been largely understudied. Our data indicate that phosphorylated cortactin tethers $\mathrm{Abl} 2$, via its $\mathrm{SH} 2$ domain, in spines and this interaction is essential in maintaining the stable actin pool (Fig. 7A). Upon loss of Abl2 or mutational disruption of phospho-tyrosine residues on cortactin, cortactin is lost from small dendritic spines and becomes enriched in a subset of large spines that retain the stable actin pool. Together, our data strongly support a model in which Abl2:cortactin interactions are critical for maintenance of the spine stable actin pool.

Our data also reveal interesting crosstalk between synaptic activity and the regulation of spine stability via the stable actin pool. Tonic enhancement of synaptic activity in Abl2-deficient neurons protects against cortactin depletion from spines and net spine loss. This observation points to a key activity-dependent mechanism that promotes cortactin enrichment in spines to stabilize them (Fig. 7B). Gradual spine loss over days in Abl2-deficient neurons is accompanied by an enlargement of a subset of remaining spines (Y. C. Lin et al., 2013; present study). Disrupting activity, with TTX, in Abl2 KD neurons does not protect against spine loss, but it blocks enlargement, again pointing to an activity-driven process for enriching cortactin in spines. We note that the residual spines that remain on cortactin $\mathrm{KD}$ neurons do not enlarge, suggesting a direct role for cortactin in spine enlargement. These observations identify the stable actin pool as a key target of activity-mediated control of net spine size and stability.

\section{References}

Adrian M, Kusters R, Storm C, Hoogenraad CC, Kapitein LC (2017) Probing the interplay between dendritic spine morphology and membrane-bound diffusion. Biophys J 113:2261-2270.

Alvarez VA, Sabatini BL (2007) Anatomical and physiological plasticity of dendritic spines. Annu Rev Neurosci 30:79-97.

Borczyk M, Śliwińska MA, Caly A, Bernas T, Radwanska K (2019) Neuronal plasticity affects correlation between the size of dendritic spine and its postsynaptic density. Sci Rep 9:1693.

Borgdorff AJ, Choquet D (2002) Regulation of AMPA receptor lateral movements. Nature 417:649-653.

Bosch M, Castro J, Saneyoshi T, Matsuno H, Sur M, Hayashi Y (2014) Structural and molecular remodeling of dendritic spine substructures during long-term potentiation. Neuron 82:444-459.

Bravo-Cordero JJ, Oser M, Chen X, Eddy R, Hodgson L, Condeelis J (2011) A novel spatiotemporal RhoC activation pathway locally regulates cofilin activity at invadopodia. Curr Biol 21:635-644.

Capani F, Martone ME, Deerinck TJ, Ellisman MH (2001) Selective localization of high concentrations of F-actin in subpopulations of dendritic spines in rat central nervous system: a three-dimensional electron microscopic study. J Comp Neurol 435:156-170.

Chazeau A, Giannone G (2016) Organization and dynamics of the actin cytoskeleton during dendritic spine morphological remodeling. Cell Mol Life Sci 73:3053-3073.
Chen YK, Hsueh YP (2012) Cortactin-binding protein 2 modulates the mobility of cortactin and regulates dendritic spine formation and maintenance. J Neurosci 32:1043-1055.

Courtemanche N, Gifford SM, Simpson MA, Pollard TD, Koleske AJ (2015) Abl2/Abl-related gene stabilizes actin filaments, stimulates actin branching by actin-related protein $2 / 3$ complex, and promotes actin filament severing by cofilin. J Biol Chem 290:4038-4046.

Deller T, Korte M, Chabanis S, Drakew A, Schwegler H, Stefani GG, Zuniga A, Schwarz K, Bonhoeffer T, Zeller R, Frotscher M, Mundel P (2003) Synaptopodin-deficient mice lack a spine apparatus and show deficits in synaptic plasticity. Proc Natl Acad Sci USA 100:10494-10499.

Fiala JC, Spacek J, Harris KM (2002) Dendritic spine pathology: cause or consequence of neurological disorders? Brain Res Brain Res Rev 39:29-54.

Fischer M, Kaech S, Knutti D, Matus A (1998) Rapid actin-based plasticity in dendritic spines. Neuron 20:847-854.

Frost NA, Shroff H, Kong H, Betzig E, Blanpied TA (2010) Single-molecule discrimination of discrete perisynaptic and distributed sites of actin filament assembly within dendritic spines. Neuron 67:86-99.

Fukazawa Y, Saitoh Y, Ozawa F, Ohta Y, Mizuno K, Inokuchi K (2003) Hippocampal LTP is accompanied by enhanced F-actin content within the dendritic spine that is essential for late LTP maintenance in vivo. Neuron 38:447-460.

Gifford SM, Liu W, Mader CC, Halo TL, Machida K, Boggon TJ, Koleske AJ (2014) Two amino acid residues confer different binding affinities of Abelson family kinase SRC homology 2 domains for phosphorylated cortactin. J Biol Chem 289:19704-19713.

Hering H, Sheng M (2003) Activity-dependent redistribution and essential role of cortactin in dendritic spine morphogenesis. J Neurosci 23:1175911769 .

Holbro N, Grunditz A, Oertner TG (2009) Differential distribution of endoplasmic reticulum controls metabotropic signaling and plasticity at hippocampal synapses. Proc Natl Acad Sci USA 106:15055-15060.

Honkura N, Matsuzaki M, Noguchi J, Ellis-Davies GC, Kasai H (2008) The subspine organization of actin fibers regulates the structure and plasticity of dendritic spines. Neuron 57:719-729.

Hotulainen P, Hoogenraad CC (2010) Actin in dendritic spines: connecting dynamics to function. J Cell Biol 189:619-629.

Iki J, Inoue A, Bito H, Okabe S (2005) Bi-directional regulation of postsynaptic cortactin distribution by BDNF and NMDA receptor activity. Eur J Neurosci 22:2985-2994.

Kerr JM, Blanpied TA (2012) Subsynaptic AMPA receptor distribution is acutely regulated by actin-driven reorganization of the postsynaptic density. J Neurosci 32:658-673.

Kim CH, Lisman JE (1999) A role of actin filament in synaptic transmission and long-term potentiation. J Neurosci 19:4314-4324.

Kim K, Lakhanpal G, Lu HE, Khan M, Suzuki A, Hayashi MK, Narayanan R, Luyben TT, Matsuda T, Nagai T, Blanpied TA, Hayashi Y, Okamoto K (2015) A temporary gating of actin remodeling during synaptic plasticity consists of the interplay between the kinase and structural functions of CaMKII. Neuron 87:813-826.

Koskinen M, Hotulainen P (2014) Measuring F-actin properties in dendritic spines. Front Neuroanat 8:74.

Koskinen M, Bertling E, Hotulainen P (2012) Methods to measure actin treadmilling rate in dendritic spines. Methods Enzymol 505:47-58.

Koskinen M, Bertling E, Hotulainen R, Tanhuanpaa K, Hotulainen P (2014) Myosin IIb controls actin dynamics underlying the dendritic spine maturation. Mol Cell Neurosci 61:56-64.

Krucker T, Siggins GR, Halpain S (2000) Dynamic actin filaments are required for stable long-term potentiation (LTP) in area CA1 of the hippocampus. Proc Natl Acad Sci USA 97:6856-6861.

Lapetina S, Mader CC, Machida K, Mayer BJ, Koleske AJ (2009) Arg interacts with cortactin to promote adhesion-dependent cell edge protrusion. J Cell Biol 185:503-519.

Lin WH, Webb DJ (2009) Actin and actin-binding proteins: masters of dendritic spine formation, morphology, and function. Open Neurosci J 3:5466.

Lin YC, Yeckel MF, Koleske AJ (2013) Abl2/Arg controls dendritic spine and dendrite arbor stability via distinct cytoskeletal control pathways. J Neurosci 33:1846-1857.

Liu W, MacGrath SM, Koleske AJ, Boggon TJ (2012) Lysozyme contamination facilitates crystallization of a heterotrimeric cortactin-Arg-lysozyme complex. Acta Crystallogr Sect F Struct Biol Cryst Commun 68:154-158. 
Luo L (2002) Actin cytoskeleton regulation in neuronal morphogenesis and structural plasticity. Annu Rev Cell Dev Biol 18:601-635.

MacGillavry HD, Kerr JM, Kassner J, Frost NA, Blanpied TA (2016) Shankcortactin interactions control actin dynamics to maintain flexibility of neuronal spines and synapses. Eur J Neurosci 43:179-193.

MacGrath SM, Koleske AJ (2012a) Cortactin in cell migration and cancer at a glance. J Cell Sci 125:1621-1626.

MacGrath SM, Koleske AJ (2012b) Arg/Abl2 modulates the affinity and stoichiometry of binding of cortactin to F-actin. Biochemistry 51:6644-6653.

Martinez-Quiles N, Ho HY, Kirschner MW, Ramesh N, Geha RS (2004) Erk/ Src phosphorylation of cortactin acts as a switch on-switch off mechanism that controls its ability to activate N-WASP. Mol Cell Biol 24:52695280.

Matus A (2000) Actin-based plasticity in dendritic spines. Science 290:754758 .

Mikhaylova M, Bar J, van Bommel B, Schatzle P, YuanXiang P, Raman R, Hradsky J, Konietzny A, Loktionov EY, Reddy PP, Lopez-Rojas J, Spilker C, Kobler O, Raza SA, Stork O, Hoogenraad CC, Kreutz MR (2018) Caldendrin directly couples postsynaptic calcium signals to actin remodeling in dendritic spines. Neuron 97:1110-1125.e1114.

Miller AL, Wang Y, Mooseker MS, Koleske AJ (2004) The Abl-related gene (Arg) requires its F-actin-microtubule cross-linking activity to regulate lamellipodial dynamics during fibroblast adhesion. J Cell Biol 165:407419.

Miller MM, Lapetina S, MacGrath SM, Sfakianos MK, Pollard TD, Koleske AJ (2010) Regulation of actin polymerization and adhesion-dependent cell edge protrusion by the Abl-related gene (Arg) tyrosine kinase and NWASp. Biochemistry 49:2227-2234.

Nagerl UV, Willig KI, Hein B, Hell SW, Bonhoeffer T (2008) Live-cell imaging of dendritic spines by STED microscopy. Proc Natl Acad Sci USA 105:18982-18987.

Okamoto K, Nagai T, Miyawaki A, Hayashi Y (2004) Rapid and persistent modulation of actin dynamics regulates postsynaptic reorganization underlying bidirectional plasticity. Nat Neurosci 7:1104-1112.

Peacock JG, Couch BA, Koleske AJ (2010) The Abl and Arg non-receptor tyrosine kinases regulate different zones of stress fiber, focal adhesion, and contractile network localization in spreading fibroblasts. Cytoskeleton (Hoboken) 67:666-675.

Peacock JG, Miller AL, Bradley WD, Rodriguez OC, Webb DJ, Koleske AJ (2007) The Abl-related gene tyrosine kinase acts through p190RhoGAP to inhibit actomyosin contractility and regulate focal adhesion dynamics upon adhesion to fibronectin. Mol Biol Cell 18:3860-3872.

Penzes P, Cahill ME, Jones KA, VanLeeuwen JE, Woolfrey KM (2011) Dendritic spine pathology in neuropsychiatric disorders. Nat Neurosci 14:285-293.

Racz B, Weinberg RJ (2004) The subcellular organization of cortactin in hippocampus. J Neurosci 24:10310-10317.
Racz B, Weinberg RJ (2006) Spatial organization of cofilin in dendritic spines. Neuroscience 138:447-456.

Racz B, Weinberg RJ (2008) Organization of the Arp2/3 complex in hippocampal spines. J Neurosci 28:5654-5659.

Rosenmund C, Westbrook GL (1993) Calcium-induced actin depolymerization reduces NMDA channel activity. Neuron 10:805-814.

Rubinson DA, Dillon CP, Kwiatkowski AV, Sievers C, Yang L, Kopinja J, Rooney DL, Zhang M, Ihrig MM, McManus MT, Gertler FB, Scott ML, Van Parijs L (2003) A lentivirus-based system to functionally silence genes in primary mammalian cells, stem cells and transgenic mice by RNA interference. Nat Genet 33:401-406.

Scherer AN, Anand NS, Koleske AJ (2018) Cortactin stabilization of actin requires actin-binding repeats and linker, is disrupted by specific substitutions, and is independent of nucleotide state. J Biol Chem 293:1302213032.

Schnoor M, Stradal TE, Rottner K (2018) Cortactin: cell functions of a multifaceted actin-binding protein. Trends Cell Biol 28:79-98.

Schubert V, Dotti CG (2007) Transmitting on actin: synaptic control of dendritic architecture. J Cell Sci 120:205-212.

Sekino Y, Kojima N, Shirao T (2007) Role of actin cytoskeleton in dendritic spine morphogenesis. Neurochem Int 51:92-104.

Sfakianos MK, Eisman A, Gourley SL, Bradley WD, Scheetz AJ, Settleman J, Taylor JR, Greer CA, Williamson A, Koleske AJ (2007) Inhibition of Rho via Arg and p190RhoGAP in the postnatal mouse hippocampus regulates dendritic spine maturation, synapse and dendrite stability, and behavior. J Neurosci 27:10982-10992.

Simpson MA, Bradley WD, Harburger D, Parsons M, Calderwood DA, Koleske AJ (2015) Direct interactions with the integrin beta1 cytoplasmic tail activate the Abl2/Arg kinase. J Biol Chem 290:8360-8372.

Star EN, Kwiatkowski DJ, Murthy VN (2002) Rapid turnover of actin in dendritic spines and its regulation by activity. Nat Neurosci 5:239-246.

Stuart JR, Gonzalez FH, Kawai H, Yuan ZM (2006) c-Abl interacts with the WAVE2 signaling complex to induce membrane ruffling and cell spreading. J Biol Chem 281:31290-31297.

Wang Y, Miller AL, Mooseker MS, Koleske AJ (2001) The Abl-related gene (Arg) nonreceptor tyrosine kinase uses two F-actin-binding domains to bundle F-actin. Proc Natl Acad Sci USA 98:14865-14870.

Weed SA, Karginov AV, Schafer DA, Weaver AM, Kinley AW, Cooper JA, Parsons JT (2000) Cortactin localization to sites of actin assembly in lamellipodia requires interactions with F-actin and the Arp2/3 complex. J Cell Biol 151:29-40.

Xiao X, Levy AD, Rosenberg BJ, Higley MJ, Koleske AJ (2016) Disruption of coordinated presynaptic and postsynaptic maturation underlies the defects in hippocampal synapse stability and plasticity in Abl2/Arg-deficient mice. J Neurosci 36:6778-6791. 